\title{
Isogeometric analysis of sound propagation through laminar flow in 2-dimensional ducts
}

Nørtoft, Peter; Gravesen, Jens; Willatzen, Morten

\section{Published in:}

Computer Methods in Applied Mechanics and Engineering

Link to article, DOI:

10.1016/j.cma.2014.11.035

Publication date:

2015

Document Version

Peer reviewed version

Link back to DTU Orbit

Citation (APA):

Nørtoft, P., Gravesen, J., \& Willatzen, M. (2015). Isogeometric analysis of sound propagation through laminar flow in 2-dimensional ducts. Computer Methods in Applied Mechanics and Engineering, 284, 1098-1119. https://doi.org/10.1016/j.cma.2014.11.035

\section{General rights}

Copyright and moral rights for the publications made accessible in the public portal are retained by the authors and/or other copyright owners and it is a condition of accessing publications that users recognise and abide by the legal requirements associated with these rights.

- Users may download and print one copy of any publication from the public portal for the purpose of private study or research.

- You may not further distribute the material or use it for any profit-making activity or commercial gain

- You may freely distribute the URL identifying the publication in the public portal 


\title{
Isogeometric Analysis of Sound Propagation through Laminar Flow in 2-Dimensional Ducts
}

\author{
Peter Nørtoft ${ }^{\mathrm{a}, *}$, Jens Gravesen ${ }^{\mathrm{a}}$, Morten Willatzen ${ }^{\mathrm{b}}$ \\ ${ }^{a}$ DTU Compute, Technical University of Denmark, Matematiktorvet 303B, DK-2800 \\ Kgs. Lyngby, Denmark, Phone: +45 4525 3031, Fax: +45 45881399 \\ ${ }^{b}$ DTU Fotonik, Technical University of Denmark, Ørsteds Plads 345, DK-2800 Kgs. \\ Lyngby, Denmark, Phone: +454525 6352, Fax: +4545936581
}

\begin{abstract}
We consider the propagation of sound through a slowly moving fluid in a 2-dimensional duct. A detailed description of a flow-acoustic model of the problem using B-spline based isogeometric analysis is given. The model couples the non-linear, steady-state, incompressible Navier-Stokes equation in the laminar regime for the flow field, to a linear, time-harmonic acoustic equation in the low Mach number regime for the sound signal. B-splines are used both to represent the duct geometry and to approximate the flow and sound fields. This facilitates an exact representation of complex duct geometries, as well as high continuity approximations of state variables. Acoustic boundary conditions on artificial truncation boundaries are treated using a mode matching formulation. We validate the model against known acoustic modes for a uniform flow through a straight duct. Improved error convergence rates are found when the acoustic pressure is approximated by higher order polynomials. Based on the model, we examine how the acoustic signal varies with sound frequency, flow speed and duct geometry. A combination of duct geometry and sound frequency is identified for which the acoustic signal is particularly sensitive to the flow speed.
\end{abstract}

Keywords:

flow acoustics, isogeometric analysis, B-spline, duct, ultrasound, mode matching

\footnotetext{
*Corresponding author.

Email addresses: penn@dtu.dk (Peter Nørtoft), jgra@dtu.dk (Jens Gravesen), morwi@fotonik.dtu.dk (Morten Willatzen)
}

Preprint submitted to Computer Methods in Applied Mechanics and Engineering 


\section{Introduction}

It is well known in physics and engineering applications that wave propagation in ducts is sensitive to the duct geometry $[1,2,3]$. In particular, spatial resonances exist at certain frequencies $[4,5,6]$, and it is anticipated that disturbances may cause large variations in signal transmission at such frequencies. While these effects have been explored in quantum mechanics, solid state physics, and optics, in particular for quasi-periodic and periodic structures $[7,8,9,10,11]$, we here investigate the influence of a slow background flow on acoustic wave propagation in 2-dimensional ducts of varying height.

Acoustic wave propagation through flows in inhomogeneous ducts has been studied extensively in the literature, based on both numerical and analytical approaches, see e.g. $[12,13,14]$. Finite element methods represent a popular numerical approach, based on, e.g., the convected Helmholtz equation [15, 16], the linearized Euler equations [17], or the so-called Galbrun's equation $[18,19,20]$. These methods are often discretized using Lagrange elements with $C^{0}$-continuity of the state variable approximations.

The current work contributes to the field of numerical methods for sound propagation through flow in ducts in two ways. Firstly, we construct a simple one-way coupled flow-acoustic model, where "one-way coupled" reflects that the flow field affects the acoustic field, but not vice versa. This is a good approximation for low-intensity acoustic excitations. The procedure we follow is first to compute the background flow based on the steady-state, incompressible Navier-Stokes equations in the laminar regime, i.e., at low Reynolds numbers, and then adobt a linear, time-harmonic flow-acoustic approach for the sound wave propagation in the low Mach number regime, using the output of the flow model as input to the acoustic model. This results in a single equation in the acoustic pressure, linear in both the flow field itself and its gradient. The low Mach number assumption corresponds to neglecting quadratic and higher order terms in the flow for the acoustic analysis.

Secondly, we base our calculations on isogeometric analysis as numerical methods. This unites the powers of finite element methods to solve partial differential equations with the powers of computer aided design (CAD) to represent complex shapes [21, 22]. A key feature of this approach is to use (variations of) B-splines, the building blocks of many CAD models, 
both to represent geometry and to approximate state variables. This allows for simple descriptions of complex duct geometries without any approximations, and provides high degrees of smoothness for both flow and acoustic fields with attractive error convergence properties. Another important feature of the method is that the acoustic eigenvalues and modes that enter the boundary conditions on artificial truncation boundaries in the proposed mode matching formulation, are represented very well with B-splines [23]. Through a consistent choice of B-splines to approximate the acoustic modes on the boundary and the acoustic field on the interior, a smooth and seamless model is obtained that requires no specification of parameters like the absorption coefficient needed in the perfectly matched layer (PML) formulation. The applicability of isogeometric analysis is well documented for fluid mechanics, see, e.g., [24, 25, 26, 27], wave phenomena and structural vibrations $[23,28,29]$, shock hydrodynamics [30], and acoustics based on the boundary element method [31].

The first aim of the paper is to establish and validate the proposed flowacoustic model for time-harmonic sound propagation at low Mach numbers through a stationary, incompressible background flow at low Reynolds numbers in 2-dimensional ducts using B-spline based isogeometric analysis. The second aim of the paper is to use the model to numerically examine how the geometry of the duct influences the sound propagation, and in particular its effect on how the acoustic signal depends on flow speed. The generic problem is sketched in Fig. 1. We consider a transmitting ultrasonic transducer mounted on the wall of a narrow duct in which we have a laminar flow of, say, air, and we examine how the acoustic signal depends on duct geometry, sound frequency, and flow speed. The exact representation of complex shapes in few degrees of freedom, and the smooth approximations of state variables embedded in the isogeometric method, make it ideal in this context.

The outline of the rest of the paper is as follows: Section 2 introduces the governing equations and approximations leading to the flow-acoustic model. The isogeometric method is presented in Section 3, after which the method is validated in Section 4 based on acoustic duct modes. Numerical results are presented in Section 5, and finally conclusions are summarized in Section 6. 


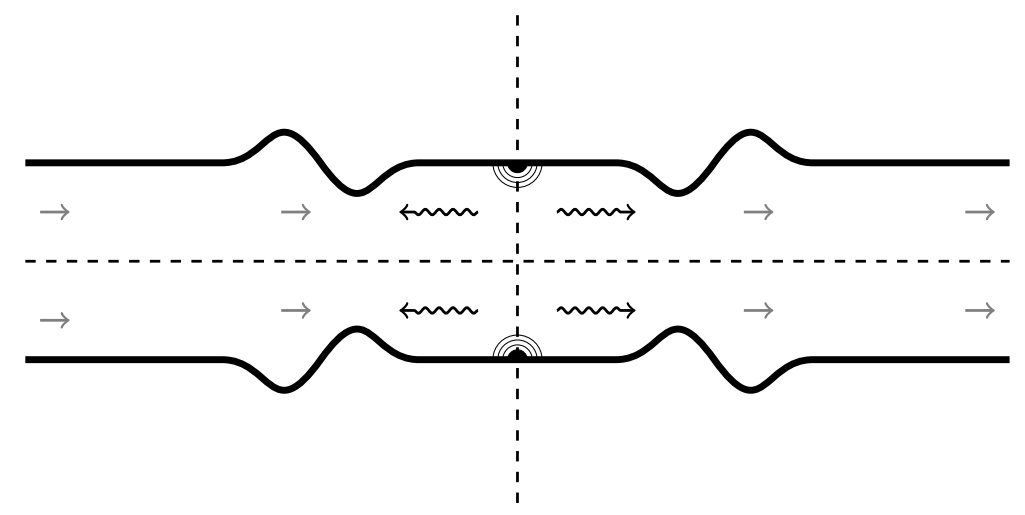

Figure 1: Sound propagates (black arrows) from acoustic sources (black rings) through a moving fluid (gray arrows) in a symmetric (dashed lines) 2-dimensional duct (solid lines).

\section{Governing Equations and Approximations}

We consider the propagation of sound through a moving fluid in a symmetric 2-dimensional duct as depicted in Fig. 1. The state of the fluid is characterised by the velocity $\mathbf{u}=(u, v)$, the pressure $p$, and the density $\rho$, assuming the fluid is isothermal, i.e., at constant temperature. These state variables are governed by the Navier-Stokes and mass continuity equations:

$$
\begin{aligned}
\rho \frac{\partial \mathbf{u}}{\partial t}+\rho(\mathbf{u} \cdot \nabla) \mathbf{u}+\nabla p-\nabla \cdot \mathbb{T} & =\mathbf{0}, \\
\frac{\partial \rho}{\partial t}+\nabla \cdot(\rho \mathbf{u}) & =0,
\end{aligned}
$$

where $t$ denotes time, and $\mathbb{T}$ is the deviatoric stress tensor.

The problem involves two distinct physical phenomena: the flow of the fluid and the propagation of sound in it. We express this separation through the state variables:

$$
\mathbf{u}=\mathbf{u}_{0}+\mathbf{u}^{\prime}, \quad p=p_{0}+p^{\prime}, \quad \rho=\rho_{0}+\rho^{\prime},
$$

where $\mathbf{u}_{0}, p_{0}$, and $\rho_{0}$ relate to the large scale mean flow, and $\mathbf{u}^{\prime}, p^{\prime}$, and $\rho^{\prime}$ relate to the small scale acoustic disturbances. For simplicity, we will assume a one-way coupling of these phenomena, such that flow phenomena $\left(\mathbf{u}_{0}, p_{0}\right.$, and $\left.\rho_{0}\right)$ influence acoustic phenomena $\left(\mathbf{u}^{\prime}, p^{\prime}\right.$, and $\left.\rho^{\prime}\right)$, but not the other way around. Hence, we may treat the flow as independent of the acoustics, and use the output of the flow model as input to the acoustic model. 


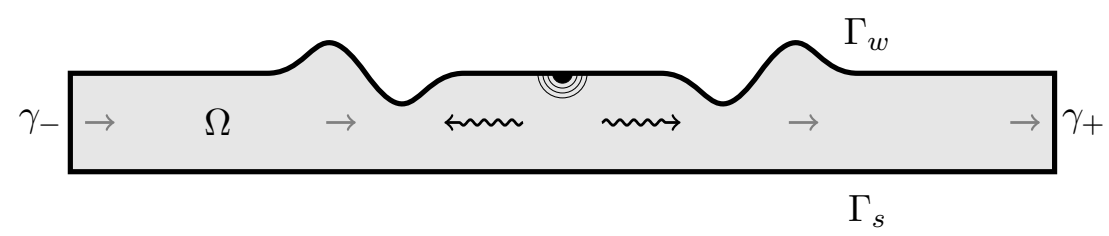

Figure 2: Model domain (light gray) and boundaries (black lines).

In the following, we consider the flow and acoustic equations over a symmetric, finite segment of one half of the symmetric, infinite 2-dimensional duct as depicted in Fig. 2. The four boundaries of the model domain $\Omega$ are: the hard wall boundary $\Gamma_{w}$, the symmetry boundary $\Gamma_{s}$, and the two artificial truncation boundaries $\gamma_{-}$and $\gamma_{+}$.

\subsection{Flow Equations}

For the flow model, we assume that the fluid is Newtonian and incompressible, and that the flow is stationary. The governing equations (1) over $\Omega$ then simplify to:

$$
\begin{aligned}
\rho_{0}\left(\mathbf{u}_{0} \cdot \nabla\right) \mathbf{u}_{0}+\nabla p_{0}-\mu \nabla^{2} \mathbf{u}_{0} & =\mathbf{0} \\
\nabla \cdot \mathbf{u}_{0} & =0
\end{aligned}
$$

where $\mu$ is the dynamic viscosity of the fluid.

The boundary conditions are:

$$
\begin{aligned}
\mathbf{u}_{0} & =\mathbf{0} & & \text { on } \Gamma_{w}, \\
v_{0}=0 \wedge \nabla u_{0} \cdot \mathbf{n} & =0 & & \text { on } \Gamma_{s}, \\
\mathbf{u}_{0} & =\mathbf{u}_{0}^{*} & & \text { on } \gamma_{-}, \\
\left(\mu \nabla\left(\mathbf{u}_{0} \cdot \mathbf{e}_{i}\right)-p \mathbf{e}_{i}\right) \cdot \mathbf{n} & =0 & & \text { on } \gamma_{+} \text {for } i=1,2,
\end{aligned}
$$

where $\mathbf{n}$ is the outward unit normal, and $\mathbf{e}_{i}$ is the $i^{\text {th }}$ unit vector. Along the hard wall boundary $\Gamma_{w}$, no-slip boundary conditions are assumed, while Eq. $(4 \mathrm{~b})$ is the symmetry condition along the symmetry edge $\Gamma_{s}$. On the flow inlet boundary $\gamma_{-}, \mathbf{u}_{0}^{*}$ prescribes a purely horizontal, parabolic velocity profile, while Eqs. (4d) prescribes the outflow condition along the outflow boundary $\gamma_{+}$. We shall refer to the hard wall boundary $\Gamma_{w}$ and the inflow boundary $\gamma_{-}$, along which the background flow velocity is explicitly prescribed, commonly as the Dirichlet boundary. 
We solve the weak, or variational, form of the system (3)-(4) which reads: given $\rho_{0}$ and $\mu$, find $u_{0} \in H_{u_{0}^{*}}^{1}(\Omega), v_{0} \in H_{v_{0}^{*}}^{1}(\Omega)$, and $p \in H^{1}(\Omega)$ such that

$$
\begin{array}{r}
\iint_{\Omega}\left(\mathcal{U} \rho_{0}\left(\mathbf{u}_{0} \cdot \nabla\right) u_{0}-p_{0} \frac{\partial \mathcal{U}}{\partial x}+\mu \nabla \mathcal{U} \cdot \nabla u_{0}\right) \mathrm{d} A=0, \\
\iint_{\Omega}\left(\mathcal{V} \rho_{0}\left(\mathbf{u}_{0} \cdot \nabla\right) v_{0}-p_{0} \frac{\partial \mathcal{V}}{\partial y}+\mu \nabla \mathcal{V} \cdot \nabla v_{0}\right) \mathrm{d} A=0, \\
\iint_{\Omega} \mathcal{P}\left(\nabla \cdot \mathbf{u}_{0}\right) \mathrm{d} A=0,
\end{array}
$$

for all functions $\mathcal{U} \in H_{0}^{1}(\Omega), \mathcal{V} \in H_{0}^{1}(\Omega)$ and $\mathcal{P} \in H^{1}(\Omega)$. Here, $H^{s}(\Omega)$ denotes the Sobolev space $H^{s}(\Omega)=\left\{f \in L^{2}(\Omega): \forall|\alpha| \leq s, \partial_{x}^{\alpha} f \in L^{2}(\Omega)\right\}$, where $\alpha=\left(\alpha_{1}, \alpha_{2}\right),|\alpha|=\alpha_{1}+\alpha_{2}$ and $\partial_{x}^{\alpha} f=\partial_{x_{1}}^{\alpha_{1}} \partial_{x_{2}}^{\alpha_{2}} f$, and $H_{g}^{s}(\Omega)$ denotes the space $H_{g}^{s}(\Omega)=\left\{f \in H^{s}(\Omega):\left.f\right|_{\Gamma_{D}}=g\right\}$, where $\Gamma_{D}$ is the part of the boundary $\partial \Omega$ where $f$ is prescribed by $g$ through a Dirichlet boundary condition.

\subsection{Acoustic Equations}

Below, we give an outline of the derivation of the governing equation for the acoustic model. The full derivation is included in Appendix A. The acoustic model sets out from the Navier-Stokes and mass conservation equations (1). By neglecting viscous effects, inserting the conventions (2) and the flow equations (3), linearizing with respect to the acoustic variables, assuming the speed-of-sound $c$ in the fluid to be constant, using the relation $\frac{D p}{D t}=c^{2} \frac{D \rho}{D t}$, where $\frac{D}{D t}=\frac{\partial}{\partial t}+(\mathbf{u} \cdot \nabla)$ is the convective derivative, and assuming small acoustic disturbances, we arrive at the following linearized acoustic equations:

$$
\begin{aligned}
\frac{\partial u^{\prime}}{\partial t}+\left(\mathbf{u}_{0} \cdot \nabla\right) u^{\prime}+\left(\mathbf{u}^{\prime} \cdot \nabla\right) u_{0}+\frac{1}{\rho_{0}} \frac{\partial p^{\prime}}{\partial x} & =0 \\
\frac{\partial v^{\prime}}{\partial t}+\left(\mathbf{u}_{0} \cdot \nabla\right) v^{\prime}+\left(\mathbf{u}^{\prime} \cdot \nabla\right) v_{0}+\frac{1}{\rho_{0}} \frac{\partial p^{\prime}}{\partial y} & =0 \\
\frac{\partial p^{\prime}}{\partial t}+\mathbf{u}_{0} \cdot \nabla p^{\prime}+\rho_{0} c^{2} \nabla \cdot \mathbf{u}^{\prime} & =0
\end{aligned}
$$

Applying separation-of-variables and assuming time-harmonic conditions, we seek acoustic solutions $\mathbf{u}^{\prime}$ and $p^{\prime}$ to Eqs. (6) of the following form:

$$
\mathbf{u}^{\prime}(t, \mathbf{x})=e^{-i \omega t} \tilde{\mathbf{u}}(\mathbf{x}), \quad p^{\prime}(t, \mathbf{x})=e^{-i \omega t} \tilde{p}(\mathbf{x}) .
$$


where $\omega$ denotes the acoustic angular frequency. Using the above assumptions, neglecting quadratic or higher order terms in the background flow $\mathbf{u}_{0}$ and its derivatives, and introducing an acoustic source $g$, the following acoustic equation can be derived:

$$
\nabla^{2} \tilde{p}+k^{2} \tilde{p}+\frac{2 i}{\omega}\left(k^{2} \mathbf{u}_{0} \cdot \nabla \tilde{p}-\frac{\partial \mathbf{u}_{0}}{\partial x} \cdot \nabla \frac{\partial \tilde{p}}{\partial x}-\frac{\partial \mathbf{u}_{0}}{\partial y} \cdot \nabla \frac{\partial \tilde{p}}{\partial y}\right)=g
$$

where $k:=\omega / c$ is the wave number.

Eq. (8) is a second order partial differential equation in the spatial part $\tilde{p}$ of the acoustic pressure disturbance, with given angular frequency $\omega$, speed of sound $c$, and background flow field $\mathbf{u}_{0}$. It corresponds to the more general Pridmore-Brown equation [32] in the limit of small Mach numbers $M:=\|\mathbf{u}\| / c \ll 1$. We note that the terms in the parenthesis relate to the background flow $\mathbf{u}_{0}$ and its gradient $\nabla \mathbf{u}_{0}$. When the background flow vanishes, the equation reduces to the usual inhomogeneous Helmholtz equation. Among the list of assumptions and approximations mentioned above, we emphasize in particular that second or higher order effects in the background flow have been neglected, which limits the equation's applicability to low Mach number flows $M \ll 1$.

The boundary conditions on the hard wall and the symmetry edge are:

$$
\nabla \tilde{p} \cdot \mathbf{n}=0 \quad \text { on } \Gamma_{w} \cup \Gamma_{s} .
$$

The treatment of the artificial truncation boundaries $\gamma_{ \pm}$is less trivial $[33,15$, 16]. Here, waves must be allowed to propagate out of the domain, and, at the same time, reflections back into the domain must be avoided. We treat the truncation boundaries using a mode matching formulation [34], based on a modal decomposition of the acoustic signal on the boundary. The crux of the mode matching method is to assume that the acoustic pressure on the truncation boundaries $\gamma_{ \pm}$may be approximated as linear combinations of so-called acoustic duct modes that propagate out of the domain. This establishes the following relations for the acoustic pressure and its normal derivative:

$$
\begin{array}{rlr}
\tilde{p}(x, y) & =\sum_{n=0}^{N_{m}^{ \pm}-1} w_{n}^{ \pm} \phi_{n}^{ \pm}(y):=\tilde{p}_{ \pm} & \text {on } \gamma_{ \pm}, \\
\nabla \tilde{p}(x, y) \cdot \mathbf{n}= \pm \frac{\partial \tilde{p}}{\partial x}(x, y)= \pm \sum_{n=0}^{N_{m}^{ \pm}-1} \lambda_{n} w_{n}^{ \pm} \phi_{n}^{ \pm}(y) & \text { on } \gamma_{ \pm}
\end{array}
$$


where $\phi_{n}$ are the acoustic duct modes, $\lambda_{n}$ are the associated (complex and signed) wave numbers, $w_{n}$ are the expansion coefficients, and $N_{m}$ is the finite (and small) number of modes.

The mode matching method involves two steps. In the first step, the acoustic duct modes, i.e., the functions $\phi_{n}$ and the wave numbers $\lambda_{n}$, are determined. For this, we assume that the truncation boundaries $\gamma_{ \pm}$are placed far away from acoustic sources and geometric ondulations, such that the background flow is independent of $x$ and normal to $\gamma_{ \pm}$, i.e., $\mathbf{u}_{0}(x, y)=\left(u_{0}(y), 0\right)$. We then write the weak form of the governing equation (8), evaluated on the truncation boundaries: given $\omega, k$, and $u_{0}$, find $\tilde{p}_{ \pm} \in H^{2}\left(\gamma_{ \pm}\right)$such that

$$
\int_{\gamma_{ \pm}}\left(\tilde{\mathcal{P}}\left[\frac{\partial^{2} \tilde{p}_{ \pm}}{\partial x^{2}}+k^{2} \tilde{p}_{ \pm}+\frac{2 i}{\omega}\left(k^{2} u_{0} \frac{\partial \tilde{p}_{ \pm}}{\partial x}-\frac{\mathrm{d} u_{0}}{\mathrm{~d} y} \frac{\partial^{2} \tilde{p}_{ \pm}}{\partial x \partial y}\right)\right]-\frac{\partial \tilde{\mathcal{P}}}{\partial y} \frac{\partial \tilde{p}_{ \pm}}{\partial y}\right) \mathrm{d} y=0
$$

for all functions $\widetilde{\mathcal{P}} \in H^{1}\left(\gamma_{ \pm}\right)$. Here, we have used the homogenous Neumann boundary conditions in Eq. (9), evaluated on the truncation boundaries. As we shall see in Section 3.4.1, the modes may then be determined by solving an eigenvalue problem based on Eq. (11). We emphasize that, as a result of the assumed symmetry around $y=0$, only symmetric modes over the full duct width are allowed in this model.

In the second step, the weights $w_{n}$ of the modes on $\gamma_{ \pm}$, as well as the acoustic pressure $\tilde{p}$ over the entire domain $\Omega$ are determined. For this, we consider the weak forms of the system (8)-(9) for the acoustic pressure over the domain $\Omega$, and of the modal relation (10a) on the truncation boundaries $\gamma_{ \pm}$. These read: given $\omega, k, f$, and $\mathbf{u}_{0}$, find $\tilde{p}_{ \pm}$and $\tilde{p} \in H^{1}(\Omega)$ such that

$$
\begin{array}{r}
\int_{\gamma_{ \pm}} \tilde{\mathcal{P}}(\nabla \tilde{p} \cdot \mathbf{n}) \mathrm{d} s-\iint_{\Omega} \nabla \tilde{\mathcal{P}} \cdot \nabla \tilde{p} \mathrm{~d} A+\iint_{\Omega} \tilde{\mathcal{P}}\left[k^{2} \tilde{p}-g\right. \\
\left.+\frac{2 i}{\omega}\left(k^{2} \mathbf{u}_{0} \cdot \nabla \tilde{p}-\frac{\partial \mathbf{u}_{0}}{\partial x} \cdot \nabla \frac{\partial \tilde{p}}{\partial x}-\frac{\partial \mathbf{u}_{0}}{\partial y} \cdot \nabla \frac{\partial \tilde{p}}{\partial y}\right)\right] \mathrm{d} A=0 \\
\int_{\gamma_{ \pm}} \widetilde{\mathcal{F}}^{ \pm}\left(\tilde{p}-\tilde{p}_{ \pm}\right) \mathrm{d} s=0,
\end{array}
$$

for all test functions $\widetilde{\mathcal{F}}^{ \pm} \in L^{2}\left(\gamma_{ \pm}\right)$and $\widetilde{\mathcal{P}} \in H^{1}(\Omega)$. These equations are then solved using the Neumann boundary conditions (10b) on the truncation boundaries. 


\section{Isogeometric Analysis}

We solve the flow-acoustic problem numerically using B-spline based isogeometric analysis, building on the Galerkin method. This section gives an account of the numerical procedure. The reader is referred to, e.g., [35] for treatment of B-splines, and to, e.g., [22] for an extensive introduction to isogeometric analysis.

\subsection{B-splines}

B-splines form the building blocks of the numerical method. For later reference, we briefly revise the basic concepts of these functions.

Univariate B-splines $\mathcal{N}_{i}^{q}:[0,1] \rightarrow \mathbb{R}$ are piecewise polynomials defined recursively from a polynomial degree $q \in \mathbb{N}$ and a set of non-decreasing knots $\Xi=\left\{\xi_{1}, \ldots, \xi_{m}\right\} \in \mathbb{R}^{m}$ :

$$
\mathcal{N}_{i}^{0}(\xi)= \begin{cases}1 & \text { if } \xi_{i} \leq \xi<\xi_{i+1} \\ 0 & \text { otherwise }\end{cases}
$$

for $q=0$, and

$$
\mathcal{N}_{i}^{q}(\xi)=\frac{\xi-\xi_{i}}{\xi_{i+q}-\xi_{i}} \mathcal{N}_{i}^{q-1}(\xi)+\frac{\xi_{i+q+1}-\xi}{\xi_{i+q+1}-\xi_{i+1}} \mathcal{N}_{i+1}^{q-1}(\xi)
$$

for $q=1,2, \ldots$ with $i=1, \ldots, m-q-1$. Here, we use the unit parametric domain $\xi \in[0,1]$, and assume open knot vectors, i.e., the boundary knots have multiplicity $q+1$ with $\xi_{1}=\xi_{2}=\ldots=\xi_{q+1}=0$ and $\xi_{m}=\xi_{m-1}=\ldots=$ $\xi_{m-q}=1$.

Bivariate tensor product B-splines $\mathcal{R}_{i, j}^{q, r}:[0,1]^{2} \rightarrow \mathbb{R}$ are defined from the univariate B-splines above:

$$
\mathcal{R}_{i, j}^{q, r}(\xi, \eta)=\mathcal{N}_{i}^{q}(\xi) \mathcal{M}_{j}^{r}(\eta)
$$

where $\mathcal{N}_{i}^{q}$ is the $i^{\text {th }}$ univariate B-spline with degree $q$ and knot vector $\Xi_{1}=$ $\left\{\xi_{1}, \ldots, \xi_{m}\right\}$ in the parametric dimension $\xi$, and $\mathcal{M}_{j}^{r}$ is the $j^{\text {th }}$ univariate B-spline with degree $r$ and knot vector $\Xi_{2}=\left\{\eta_{1}, \ldots, \eta_{n}\right\}$ in the parametric dimension $\eta$. 


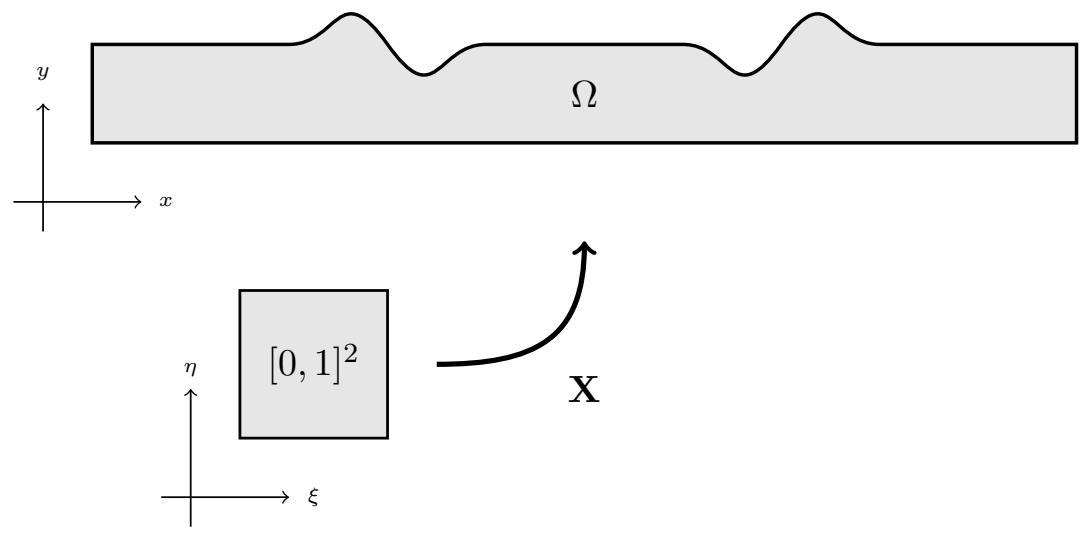

Figure 3: Parametrization of the model domain.

\subsection{Geometry Parametrization}

Using the above functions, a parametrization $\mathbf{X}:[0,1]^{2} \rightarrow \mathbb{R}^{2}$ of the computational domain is constructed, see Fig. 3:

$$
\mathbf{X}(\xi, \eta)=(x(\xi, \eta), y(\xi, \eta))=\sum_{i=1}^{N_{\text {fun }}^{g}} \underline{\mathbf{x}}_{i} \mathcal{R}_{i}^{g}(\xi, \eta),
$$

where $\mathcal{R}_{i}^{g}$ are tensor product B-splines with given polynomial degrees and knot vectors, $\underline{\mathbf{x}}_{i}$ are the associated expansion coefficients (known as control points), and $N_{\text {fun }}^{g}$ is the number of terms in the expansion.

The geometry parametrization serves as foundation for both the flow model and the acoustic model. Rather than solving the equations over the physical domain $\Omega$, we pull them back to the parameter domain $[0,1]^{2}$ and solve them there.

For later reference, the gradient $\bar{\nabla}$ and the Hessian matrix $\overline{\mathbf{H}}$ in physical space $\Omega$ of any scalar quantity $\bar{h}$ are related to their counterparts $\nabla, \mathbf{H}$, and $h$ in parameter space $[0,1]^{2}$ by the following relations:

$$
\begin{aligned}
\nabla h & =\mathbf{J}^{T} \overline{\nabla h} \\
\mathbf{H}(h) & =\mathbf{J}^{T} \overline{\mathbf{H}}(\bar{h}) \mathbf{J}+\sum_{m=1}^{2} \mathbf{H}\left(x_{m}\right) \mathbf{e}_{m}^{T} \overline{\nabla h},
\end{aligned}
$$


where $\mathbf{J}$ is the Jacobian matrix of the parametrization. These relations are easily solved for the quantities $\overline{\nabla h}$ and $\overline{\mathbf{H}}(\bar{h})$ in physical space whose elements appear in the governing equations, and expressed through the quantities $\nabla h$, $\mathbf{H}(h), \mathbf{J}, \mathbf{H}(x)$, and $\mathbf{H}(y)$ in parameter space where we solve the equations.

\subsection{Flow Model}

Approximations of the background flow velocity and pressure fields are constructed in a similar fashion as for the geometry (15):

$$
\begin{aligned}
& \mathbf{u}=\sum_{i=1}^{N_{\text {dof }}^{\mathbf{u}}} \underline{\mathbf{u}}_{i} \mathcal{R}_{i}^{\mathbf{u}}+\sum_{i=N_{\text {dof }}^{\mathbf{u}}+1}^{N_{\text {fun }}^{\mathbf{u}}} \underline{\mathbf{u}}_{i} \mathcal{R}_{i}^{\mathbf{u}}, \\
& p=\sum_{i=1}^{N_{\text {fun }}^{p}} \underline{p}_{i} \mathcal{R}_{i}^{p},
\end{aligned}
$$

where, for the $\mathbf{u}$-field, the basis functions $\mathcal{R}^{\mathbf{u}}$ are tensor product B-splines with given knot vectors and polynomial degrees, the expansions coefficients $\underline{\mathbf{u}}$ are the unknown control variables to be determined, and $N^{\mathbf{u}}$ is the number of terms in the expansion, and similarly for the $p$-field. Also, for book keeping purposes, the velocity expansion has been split into terms related to basis functions with support on the Dirichlet boundary and terms related to basis function without support on the Dirichlet boundary.

Using suitable functions $\mathcal{R}^{\mathbf{u}}$ and $\mathcal{R}^{p}$ as test functions in the weak formulation of the governing equations (5), inserting the discretizations of the state variables (17) into it, interchanging order of summation and integration, and rearranging terms, the following system of non-linear equations can be derived:

$$
\begin{aligned}
{\left[\begin{array}{ccc}
\mu \mathbf{K}_{1}+\rho_{0} \mathbf{C}_{1}(\underline{\mathbf{u}}) & \mathbf{0} & -\mathbf{G}_{1}^{T} \\
\mathbf{0} & \mu \mathbf{K}_{2}+\rho_{0} \mathbf{C}_{2}(\underline{\mathbf{u}}) & -\mathbf{G}_{2}^{T} \\
\mathbf{G}_{1} & \mathbf{G}
\end{array}\right]\left[\begin{array}{c}
\underline{\mathbf{u}}_{1} \\
\underline{\mathbf{u}}_{2} \\
\mathbf{p}
\end{array}\right] } \\
=-\left[\begin{array}{ccc}
\mu \mathbf{K}_{1}^{\star}+\rho_{0} \mathbf{C}_{1}^{\star}(\underline{\mathbf{u}}) & \mathbf{0} \\
\mathbf{0} & \mu \mathbf{K}_{2}^{\star}+\rho_{0} \mathbf{C}_{2}^{\star}(\underline{\mathbf{u}}) \\
\mathbf{G}_{1}^{\star} & \mathbf{G}_{2}^{\star}
\end{array}\right]\left[\begin{array}{l}
\underline{\mathbf{u}}_{1}^{\star} \\
\underline{\mathbf{u}}_{2}^{\star}
\end{array}\right]
\end{aligned}
$$


or simply $\mathbf{M}(\mathbf{U}) \mathbf{U}=\mathbf{F}$, with

$$
\begin{aligned}
\underline{\mathbf{K}}_{i, j, k} & =\iint_{[0,1]^{2}}{\overline{\nabla \mathcal{R}_{i}^{u_{k}}}}^{T} \overline{\nabla \mathcal{R}_{j}^{u_{k}}} \operatorname{det}(\mathbf{J}) \mathrm{d} \Xi \\
\underline{\mathbf{C}}_{i, j, k} & =\iint_{[0,1]^{2}} \mathcal{R}_{i}^{u_{k}} \mathbf{u}^{T} \overline{\nabla \mathcal{R}_{j}^{u_{k}}} \operatorname{det}(\mathbf{J}) \mathrm{d} \Xi \\
\underline{\mathbf{G}}_{i, j, k} & =\iint_{[0,1]^{2}} \mathcal{R}_{i}^{p} \mathbf{e}_{k}^{T} \overline{\nabla \mathcal{R}_{j}^{u_{k}}} \operatorname{det}(\mathbf{J}) \mathrm{d} \Xi \\
\underline{\mathbf{K}}_{k} & =\left[\begin{array}{ll}
\mathbf{K}_{k} & \mathbf{K}_{k}^{\star}
\end{array}\right] \\
\underline{\mathbf{C}}_{k} & =\left[\begin{array}{ll}
\mathbf{C}_{k} & \mathbf{C}_{k}^{\star}
\end{array}\right] \\
\underline{\mathbf{G}}_{k} & =\left[\begin{array}{ll}
\mathbf{G}_{k} & \mathbf{G}_{k}^{\star}
\end{array}\right], \\
\underline{\mathbf{u}}_{k}^{T} & =\left[\begin{array}{ll}
\underline{\mathbf{u}}_{k}^{T} & \underline{\mathbf{u}}_{k}^{\star T}
\end{array}\right],
\end{aligned}
$$

where all starred quantities relate directly to the Dirichlet boundary conditions, as described in Section 3.5 below.

\subsection{Acoustic Model}

The acoustic model involves two steps: The first step determines the acoustic duct modes on the truncation boundaries $\gamma_{ \pm}$, i.e., the functions $\phi_{n}$ and the wave numbers $\lambda_{n}$. The second step determines the weights $w_{n}$ of the modes on $\gamma_{ \pm}$, as well as the acoustic pressure $\tilde{p}$ over the entire domain $\Omega$.

\subsubsection{Acoustic Duct Modes}

To determine the acoustic duct modes, we approximate the pressure in the regions far upstream and far downstream by the following expression:

$$
\tilde{p}(x, y)=\sum_{\ell=1}^{n} \underline{\phi}_{\ell}(x) \overline{\mathcal{M}}_{\ell}(y),
$$

where $\mathcal{M}_{\ell}$ are univariate B-splines defined over the parameter domain $\xi \in[0,1]$, $\overline{\mathcal{M}}_{\ell}$ are their image in physical space, and thus functions of $y, \phi_{\ell}$ are expansion coefficients that are functions of $x$, and $n$ is the number of terms in the expansion.

By inserting the approximation (20) into the Eq. (11) on the truncation boundaries, using $\overline{\mathcal{M}}_{k}(y)$ as test functions, and pulling the integrals 
back to parameter domain $[0,1]$, the following system of ordinary differential equations is obtained:

$$
\mathbf{R} \underline{\ddot{\phi}}+\frac{2 i}{\omega} \mathbf{S} \underline{\dot{\phi}}+\mathbf{T} \underline{\phi}=\mathbf{0}
$$

where dot denotes differentiation with respect to $x$, and

$$
\begin{aligned}
\mathbf{R}_{k, \ell} & =\int_{0}^{1} \mathcal{M}_{k} \mathcal{M}_{\ell} y^{\prime} \mathrm{d} \xi \\
\mathbf{S}_{k, \ell} & =\int_{0}^{1} \mathcal{M}_{k}\left(k^{2} u_{0} \mathcal{M}_{\ell}-\frac{\mathrm{d} u_{0}}{\mathrm{~d} y} y^{\prime-1} \mathcal{M}_{\ell}^{\prime}\right) y^{\prime} \mathrm{d} \xi \\
\mathbf{T}_{k, \ell} & =\int_{0}^{1} \mathcal{M}_{k}\left(-\left(y^{\prime-1}+y^{\prime \prime} y^{\prime-2}\right) \mathcal{M}_{\ell}^{\prime}+k^{2} \mathcal{M}_{\ell}\right) y^{\prime} \mathrm{d} \xi \\
\underline{\phi} & =\left(\begin{array}{lll}
\underline{\phi}_{1}(x) & \ldots & \left.\underline{\phi}_{n}(x)\right)^{T}
\end{array}\right.
\end{aligned}
$$

for $k, \ell=1, \ldots, n$, where prime denotes differentiation with respect to $\xi$. The second order system (21) can be rewritten as the first order system

$$
\left[\begin{array}{cc}
\mathbf{I} & \mathbf{0} \\
\mathbf{0} & \mathbf{R}
\end{array}\right]\left[\begin{array}{c}
\dot{\phi} \\
\dot{\boldsymbol{\theta}}
\end{array}\right]=\left[\begin{array}{cc}
\mathbf{0} & \mathbf{I} \\
-\mathbf{T} & -\frac{2 i}{\omega} \mathbf{S}
\end{array}\right]\left[\frac{\boldsymbol{\phi}}{\underline{\boldsymbol{\theta}}}\right] .
$$

By solving the generalised eigenvalue problem

$$
\left[\begin{array}{cc}
\mathbf{0} & \mathbf{I} \\
-\mathbf{T} & -\frac{2 i}{\omega} \mathbf{S}
\end{array}\right]\left[\frac{\boldsymbol{\phi}}{\boldsymbol{\theta}}\right]=\lambda\left[\begin{array}{cc}
\mathbf{I} & \mathbf{0} \\
\mathbf{0} & \mathbf{R}
\end{array}\right]\left[\frac{\boldsymbol{\phi}}{\underline{\boldsymbol{\theta}}}\right]
$$

for a fixed value of $x$, we obtain $2 n$ pairs of eigenvalues, $\lambda_{k}$, and eigenvectors, $\left(\underline{\phi}_{k} \underline{\boldsymbol{\theta}}_{k}\right)^{T}$. Each eigenvalue represents a (complex and signed) wave number, and each eigenvector corresponds to a (complex) acoustic duct mode

$$
\phi_{k}(y)=\sum_{\ell=1}^{n} \underline{\phi}_{\ell, k} \overline{\mathcal{M}}_{\ell}(y)
$$

on the truncation boundary corresponding to the given value of $x$. Without loss of generality, we may require that $\int_{0}^{R} \phi_{k} \phi_{k}^{*} \mathrm{~d} y=1$ and $\operatorname{Im}\left(\phi_{k}(0)\right)=0$. 


\subsubsection{Acoustic Pressure}

To compute the acoustic pressure $\tilde{p}$ over the entire domain, and the weights $w_{n}$ of the modes on the truncation boundaries, we will, as for the geometry and the background flow variables, seek solutions of the following form:

$$
\tilde{p}=\sum_{i=1}^{N_{\text {fun }}^{\tilde{p}}} \underline{\tilde{p}}_{i} \mathcal{R}_{i}^{\tilde{p}}
$$

where the basis functions $\mathcal{R}^{\tilde{p}}$ are tensor product B-splines with given knot vectors and polynomial degrees, the expansions coefficients $\tilde{\boldsymbol{p}}$ are the unknown control variables to be determined, and $N_{\text {fun }}^{\tilde{p}}$ is the number of terms in the expansion.

We emphasize that the bivariate B-splines $\mathcal{R}^{\tilde{p}}$ in Eq. (26), used to approximate the pressure in the entire domain, are natural extensions of the univariate B-splines in Eq. (20) $\mathcal{M}$, used to approximate the pressure on the truncation boundaries, as expressed through Eq. (14).

As test functions in the weak formulation of the governing equations (12), we use the basis functions $\mathcal{R}^{\tilde{p}}$ and the (B-spline approximations from above of the) propagative acoustic duct modes $\phi^{ \pm}$. By inserting the acoustic field approximation (26) and the acoustic duct mode approximation (25) into these equations, exploiting the Neumann boundary condition (10b), rearranging terms and interchanging order of integration and summation, we arrive at the following system of linear equations:

$$
\left[\begin{array}{cc}
-\mathrm{D}+k^{2} \mathbf{M}+\frac{2 i}{\omega}\left(k^{2} \mathbf{L}-\mathbf{Q}\right) & \boldsymbol{\Psi} \boldsymbol{\Phi} \boldsymbol{\Lambda} \\
\boldsymbol{\Phi}^{T} \boldsymbol{\Psi} & -\boldsymbol{\Phi}^{T} \boldsymbol{\Psi} \boldsymbol{\Phi}
\end{array}\right]\left[\begin{array}{c}
\frac{\tilde{\mathbf{p}}}{\boldsymbol{w}}
\end{array}\right]=\left[\begin{array}{l}
\mathrm{g} \\
\mathbf{0}
\end{array}\right]
$$

where

$$
\begin{aligned}
\mathbf{D}_{i, j} & =\iint_{[0,1]^{2}} \overline{\nabla \mathcal{R}_{i}^{\tilde{p}}} \overline{\nabla \mathcal{R}_{j}^{\tilde{p}}} \operatorname{det}(\mathbf{J}) \mathrm{d} \Xi \\
\mathbf{M}_{i, j} & =\iint_{[0,1]^{2}} \mathcal{R}_{i}^{\tilde{p}} \mathcal{R}_{j}^{\tilde{p}} \operatorname{det}(\mathbf{J}) \mathrm{d} \Xi \\
\mathbf{L}_{i, j} & =\iint_{[0,1]^{2}} \mathcal{R}_{i}^{\tilde{p}} \mathbf{u}_{0}^{T} \overline{\nabla \mathcal{R}_{j}^{\tilde{p}}} \operatorname{det}(\mathbf{J}) \mathrm{d} \Xi \\
\mathbf{Q}_{i, j} & =\iint_{[0,1]^{2}} \mathcal{R}_{i}^{\tilde{p}} \sum_{m=1}^{2} \mathbf{e}_{m}^{T} \overline{\mathbf{H} \mathcal{R}_{j}^{\tilde{p}}} \overline{\nabla \mathbf{u}_{0}} \mathbf{e}_{m} \operatorname{det}(\mathbf{J}) \mathrm{d} \Xi,
\end{aligned}
$$




$$
\begin{aligned}
\boldsymbol{\Psi}_{i, j} & =\int_{[0,1]} \mathcal{R}_{i}^{\tilde{p}} \mathcal{R}_{j}^{\tilde{p}}\left\|\gamma^{\prime}\right\| \mathrm{d} \xi, \\
\boldsymbol{\Phi}_{i, k} & = \begin{cases}\phi_{\ell, k} & \text { if } \operatorname{supp}\left(\mathcal{R}_{i}^{\tilde{p}}\right) \cap \gamma_{ \pm} \neq \emptyset \\
0 & \text { otherwise }\end{cases} \\
\boldsymbol{\Lambda}_{k, \ell} & =\delta_{k, l} \lambda_{k}, \\
\mathbf{g}_{i} & =\iint_{[0,1]^{2}} \mathcal{R}_{i}^{\tilde{p}} g \operatorname{det}(\mathbf{J}) \mathrm{d} \Xi,
\end{aligned}
$$

for $i, j=1, \ldots, N_{\text {fun }}^{\tilde{p}}$ and $k, \ell=0, \ldots, N^{m}-1$, where $\delta_{k, l}$ is the Kronecker delta. Here, $\mathbf{L}$ is due to the flow field $\mathbf{u}_{0}$ in the interior, $\mathbf{Q}$ is due to its gradient $\nabla \mathbf{u}_{0}$ in the interior, $\boldsymbol{\Phi}$ and $\boldsymbol{\Lambda}$ depend on $\mathbf{u}_{0}$ and (the tangential component of $\nabla \mathbf{u}_{0}$ on the truncation boundaries $\gamma_{ \pm}$, while $\mathbf{D}, \mathbf{M}, \boldsymbol{\Psi}$, and $\mathrm{g}$ appear independently of the background flow.

\subsection{Implementation}

The Dirichlet boundary conditions, for which the background flow is explicitly prescribed, are enforced strongly. This is done by specifying suitable control variables $\underline{\mathbf{u}}^{*}$ corresponding to basis functions with support on the relevant boundary. With $C^{2}$ quartic basis functions to approximate the velocity field, the vanishing velocity field on the hard wall $\Gamma_{w}$ and the parabolic velocity profile on the inflow boundary $\gamma_{-}$may be enforced exactly. The Neumann boundary conditions, for which the normal component of the background flow velocity gradient or the acoustic pressure gradient are prescribed, are enforced weakly by equating the corresponding parts of the boundary integrals to their respective values.

The mode matching formulation for the acoustic truncation boundary conditions yeilds both propagative and evanescent modes, as demonstrated in section 5 below. Typically, a finite and small number of propagative modes modes are found, while the number of evanscent modes is bounded only by the numerical discretization. We base the mode matching formulation on the propagative modes, while the evanescent modes are neglected. Numerical tests in which evanescent modes were also included have indicated that these play an insignificant role on the result compared to the propagative modes.

In the numerical examples in the following sections, we will usually take $\mathcal{R}_{i}^{g}$ for the geometry parametrization as bi-quadratic tensor product B-splines. For the flow approximations, we will take $\mathcal{R}_{i}^{u}$ and $\mathcal{R}_{i}^{v}$ as bi-quartic and $\mathcal{R}_{i}^{p}$ 
as bi-cubic, respectively, all $C^{2}$ across knots cf. [27]. For the acoustic approximation, $\mathcal{R}_{i}^{\tilde{p}}$ are taken as bi-quartic, unless otherwise stated.

\section{Acoustic Duct Modes}

In this section we consider the propagation of sound waves in a uniform flow through a straight duct. The setup is sketched in Fig. 4. We emphasize that this problem is simpler than the original one described in Fig. 2, due to the trivial shape of the duct, the simple flow through it, and the lack of an interior acoustic source. These simplications, however, make the problem suitable for numerical validation of the proposed method.

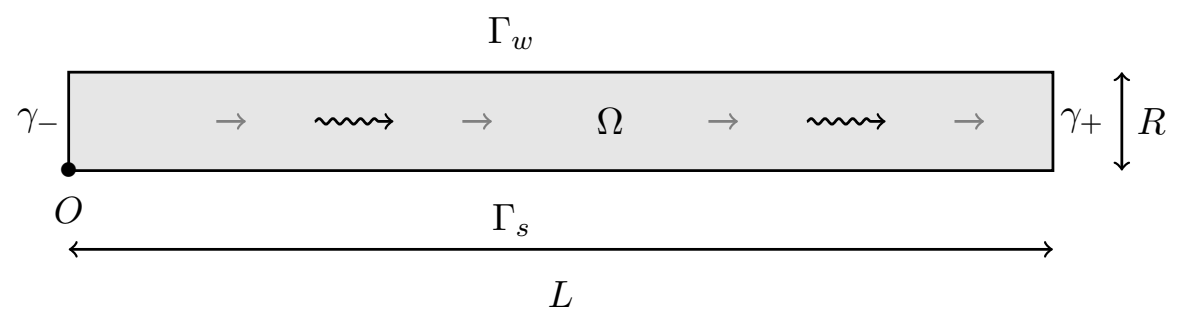

Figure 4: The modal problem.

We focus our attention on the acoustic model, and refer the reader to [27] for a validation of the flow model. Hence, we explicitly prescribe the background flow based on an artificial uniform flow profile with a given flow speed $U_{0}$ :

$$
\boldsymbol{u}_{0}=\left(u_{0}, v_{0}\right)=\left(U_{0}, 0\right) .
$$

Analytical solutions are readily available for this artificial problem, and we may validate the acoustic model numerically by examining how well the method is able to reproduce these solutions.

The following represent analytical solutions of Eq. (8) for the acoustic pressure in a straight duct of height $R$ with the uniform background flow as given in Eq. (29):

$$
\tilde{p}_{n}^{*}=A_{n} \cos \left(\alpha_{n} y\right) \exp \left(i \beta_{n} x\right)
$$


where

$$
\begin{aligned}
& A_{n}= \begin{cases}1 & \text { if } n=0, \\
\sqrt{2} & \text { otherwise }\end{cases} \\
& \alpha_{n}=n \pi / R, \\
& \beta_{n}=-k M \pm \sqrt{k^{2}\left(1+M^{2}\right)-(n \pi / R)^{2}}
\end{aligned}
$$

for non-negative integers $n \in \mathbb{N}$. This corresponds to the normalized acoustic duct modes

$$
\phi_{n}(y)=A_{n} \cos \left(\alpha_{n} y\right)
$$

with propagation constants $\beta_{n}$, travelling towards $\pm \infty$. Modes with $n \leq$ $\sqrt{1+M^{2}} k R / \pi$ are propagative, while all others are evanescent. Second order terms in the Mach number $M$ are included here to facilitate comparison with the exact modes.

\begin{tabular}{llll}
\hline \hline Parameter & Description & Value & Unit \\
\hline$\mu$ & Dynamic viscosity & $2.0 \cdot 10^{-5}$ & $\mathrm{~kg} /\left(\mathrm{m} \mathrm{s}^{3}\right.$ \\
$\rho$ & Background density & 1.2 & $\mathrm{~kg} / \mathrm{m}^{3}$ \\
$c$ & Speed-of-sound & $3.4 \cdot 10^{2}$ & $\mathrm{~m} / \mathrm{s}$ \\
$R$ & Duct height & $2.0 \cdot 10^{-2}$ & $\mathrm{~m}$ \\
$L$ & Duct length & 0.2 & $\mathrm{~m}$ \\
$f_{0}$ & Sound frequency & $3.0 \cdot 10^{4}$ & $\mathrm{~Hz}$ \\
$U_{0}$ & Flow speed & 1.0 & $\mathrm{~m} / \mathrm{s}$ \\
\hline \hline
\end{tabular}

Table 1: Parameter values for the numerical validation.

We specify numerical parameter values corresponding to the propagation of ultrasound in a laminar flow through a straight, narrow duct filled with air. The values are listed in Table 1 . With the duct height as the characteristic length, these values yield a Reynolds number of $\operatorname{Re}=\rho U_{0} R / \mu \simeq 1 \cdot 10^{3}$, a Mach number of $M=U_{0} / c \simeq 3 \cdot 10^{-3}$, a Helmholtz number of He $=\omega R / c \simeq$ 11 , and a Strouhal number of $\mathrm{St}=\omega R / U_{0} \simeq 4 \cdot 10^{3}$. The simple geometry is represented using linear B-splines.

\subsection{Acoustic Modal Decomposition}

First, we analyse how well the acoustic duct modes on the truncation boundary are computed in the first step of the mode matching method. We 
illustrate the structure of the modes computed using a cubic B-spline discretization, and we compare the quality of the modes computed using cubic and quartic discretizations based on both B-splines and on Lagrange polynomials. These bases are both polynomial but differ in terms of continuity. Across knots/elements, the B-spline discretizations are $C^{q-1}$, while the Lagrange discretizations are $C^{0}$. Since only the lowest modes enter the mode matching formulation, we focus our analysis on these, and note that higher modes might display even greater differences than those reported here [23].

The structure of the computed modes, based on a cubic univariate Bspline discretization with 32 degrees of freedom, is shown in Fig. 5. Fig. 5a shows the propagation constants $\lambda$ in the complex plane corresponding to the six lowest modes for a fixed frequency of $f=30 \mathrm{kHz}$. A given mode can be categorized as propagative or evanescent, depending on whether $\lambda$ is on or off the imaginary axis, indicating whether the mode will propagate or not. The mode may also be characterized as positive (travelling towards $+\infty$ ) or negative (travelling towards $-\infty$ ), depending on whether $\operatorname{Im}(\lambda)>-k M$ or $\operatorname{Im}(\lambda)<-k M$, or, when the imaginary part vanishes, whether $\lambda$ has a strictly positive or negative real part. Eight propagative modes are found, four in each direction, in agreement with the analytical values in Eq. (31). The number of evanescent modes is bounded only by the number of degreesof-freedom of the discretization, and only the first two in each direction are shown here. In Fig. 5b, the six modal functions $\phi$ corresponding to the six propagation constants in each direction in Fig. 5a are shown, with propagative modes drawn in solid, and evanescent modes drawn in dashed. These agree well with the analytical modes as given in Eq. (32). Finally, Figs. $5 \mathrm{c}$ and $5 \mathrm{~d}$ show the real part and the imaginary part, respectively, of the propagation constants corresponding to the six lowest positive modes for frequencies in the range $f \in[10,50] \mathrm{kHz}$. Also shown, in dashed, are the analytical cutoff frequencies within this range. As the frequency is increased from $10 \mathrm{kHz}$ to $50 \mathrm{kHz}$, the number of propagative modes grows from two to six, in agreement with the analytical expression in Eq. (31). In the following, we analyse exactly how good this agreement is.

We now analyze the quality of the modes computed by different discretizations. Fig. 6 depicts the error on the computed propagation constant for the six lowest positive modes as a function of frequency in the range $f \in[10,50] \mathrm{kHz}$. The error is evaluated based on the modulus scaled by the 

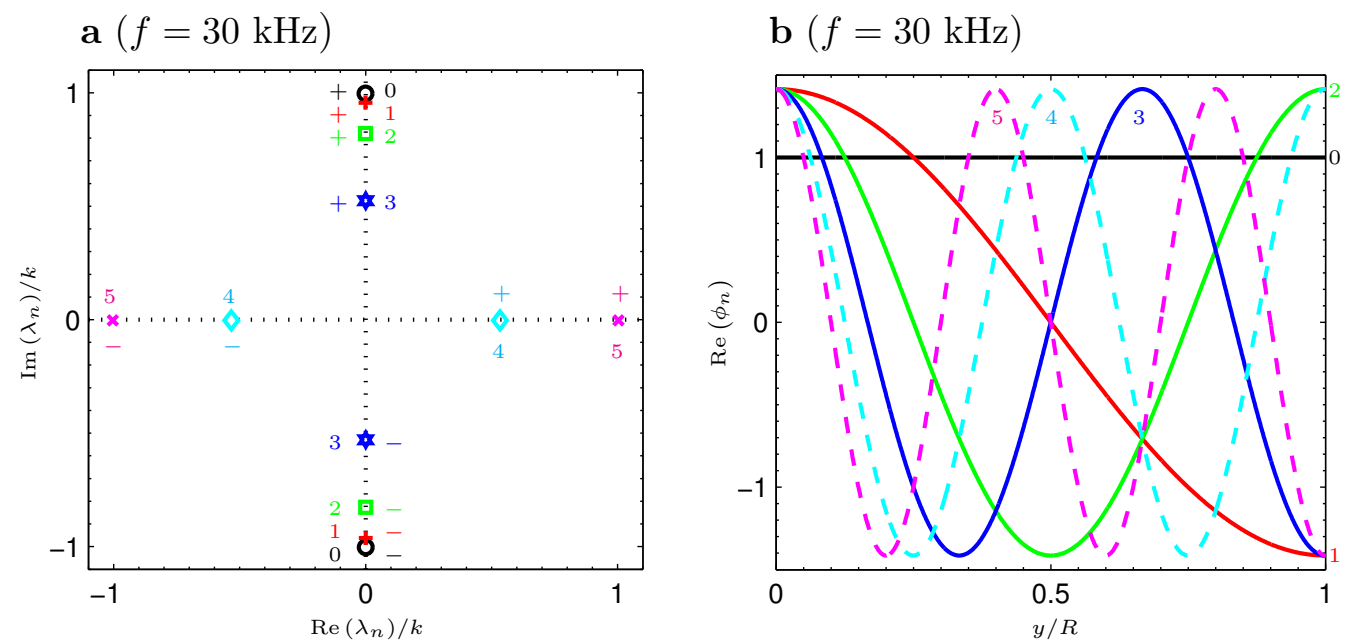

c (positive modes)

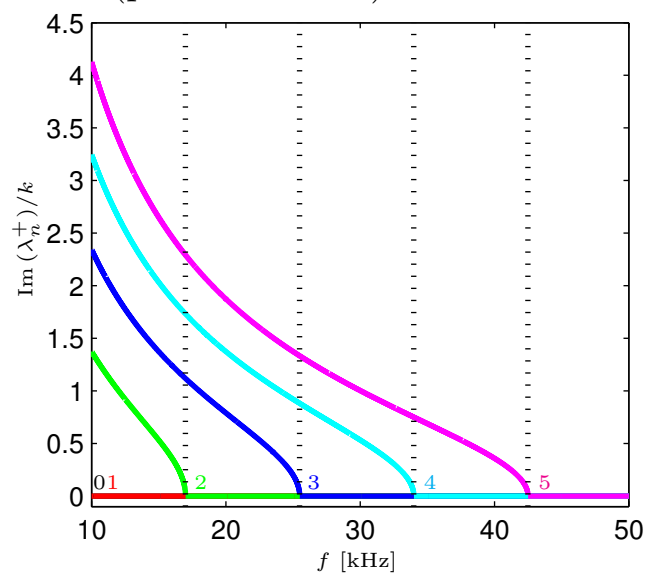

d (positive modes)

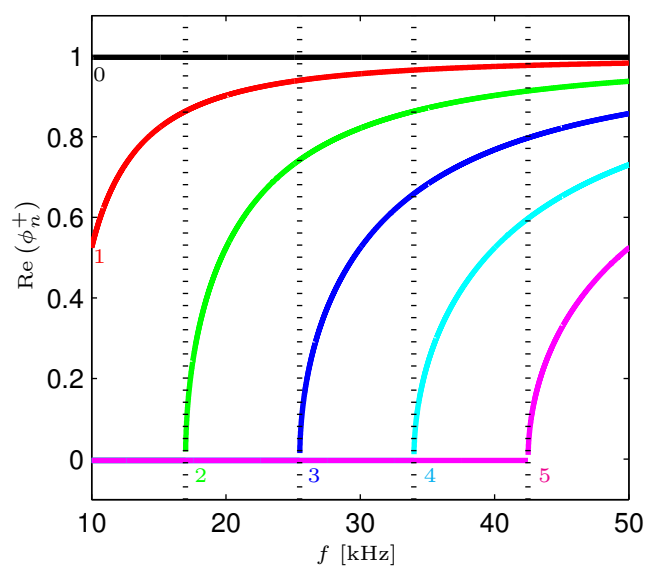

Figure 5: Computed acoustic duct modes using a cubic B-spline discretization. Propagation constants $\lambda$ (a) and real part of mode functions $\phi(\mathbf{b})$ for $f=30 \mathrm{kHz}$, and real part (c) and imaginary part (d) of the positive propagation constants as a function of frequency for $f \in[10,50] \mathrm{kHz}$.

wave number:

$$
\epsilon_{\lambda_{n}}=\frac{\left|\lambda_{n}-i \beta_{n}\right|}{k}
$$

Fig. 6a shows the error for cubic univariate B-spline and Lagrange discretizations with 32 and 34 degrees of freedom, respectively, and Fig. 6b for quartic univariate B-spline and Lagrange discretizations with 32 and 37 degrees of freedom, respectively. To avoid poluting the figure with numerical noise, er- 

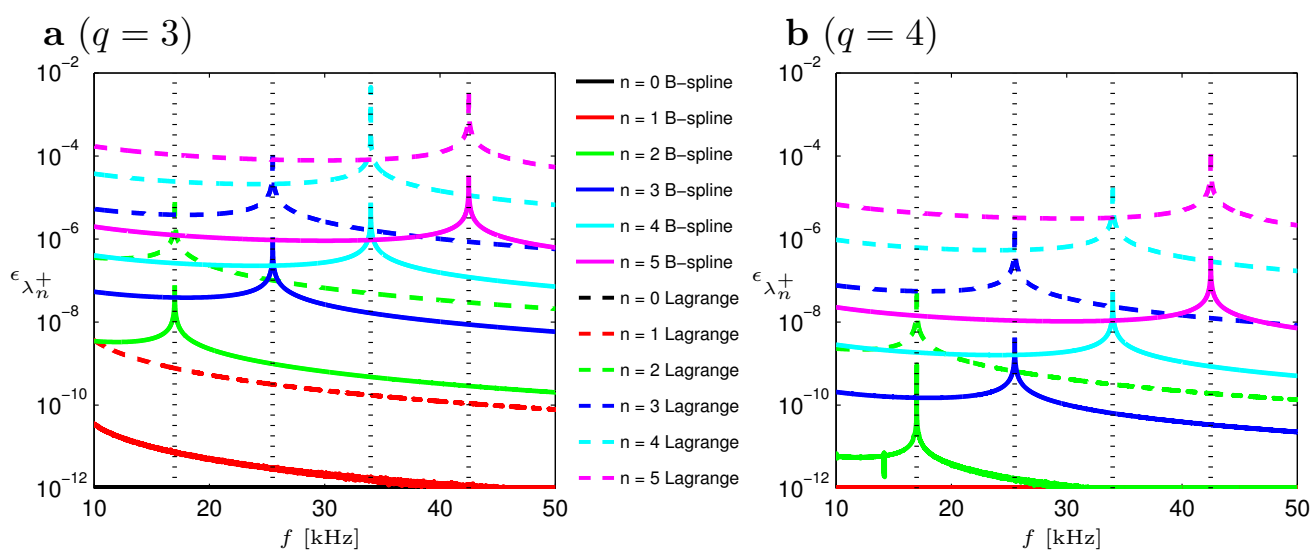

Figure 6: Error on the modulus of the computed propagation constants corresponding to the first six positive modes using cubic (a) and quartic (b) B-spline (solid lines) and Lagrange (dashed lines) discretizations.

rors smaller than $10^{-12}$ are set equal to $10^{-12}$. The cutoff frequencies stand out clearly in the figure, where errors grow by up to an order of magnitude. This pattern is associated with the vanishing of the propagation constant $\beta_{n}$ close to the transition from the evanescent state to the propagative state of a given mode $n$. For both discretization types and both polynomial degrees, we see that lower modes are better resolved. Focusing first on the two B-spline discretizations, we note that the first mode is actually determined exactly (to machine precision) in the cubic case, and the first two to three in the quartic case. For the two Lagrange discretizations, the first mode is determined exactly in the cubic case, and the first two in the quartic case. Comparing the cubic B-spline discretization with the cubic Lagrange discretizations, we see that the error for the B-spline discretization is around two orders of magnitude smaller than for the Lagrange discretization. For the quartic discretizations, this difference grows slightly. Evaluating the discretization in a per-degree-of-freedom sense, the smooth B-splines are thus favorable compared to the non-smooth Lagrange polynomials. This result aligns well with previous findings [23, 29].

\subsection{Acoustic Pressure Discretizations}

Next, we investigate how well the interior acoustic pressure, computed in the second step of the mode matching method, is approximated. We examine how different approximations of the acoustic pressure influence the overall quality of the method for a fixed frequency. Again, we compare B-splines 
with Lagrange polynomials for different polynomial degrees. To assess the quality of the method, we now use the normalised $L_{2}$-norm of the modulus of the pressure residual:

$$
\epsilon_{\tilde{p}}^{2}=\frac{\iint_{\Omega}\left|\tilde{p}-\tilde{p}^{*}\right|^{2} \mathrm{~d} A}{\iint_{\Omega}\left|\tilde{p}^{*}\right|^{2} \mathrm{~d} A} .
$$

To simplify the computational setup, we replace the mode matching boundary condition (investigated in Section 4.3 below) on the two truncation boundaries $\gamma_{ \pm}$by explicitly prescribing the acoustic pressure on these, corresponding to the $n^{\text {th }}$ positive mode. That is, we set $\tilde{p}=\tilde{p}_{n}^{*}$ on $\gamma_{ \pm}$using Eq. (32). This may be seen as a trivial instance of the mode matching method, in which we know the acoustic mode and its weight on the boundary beforehand.
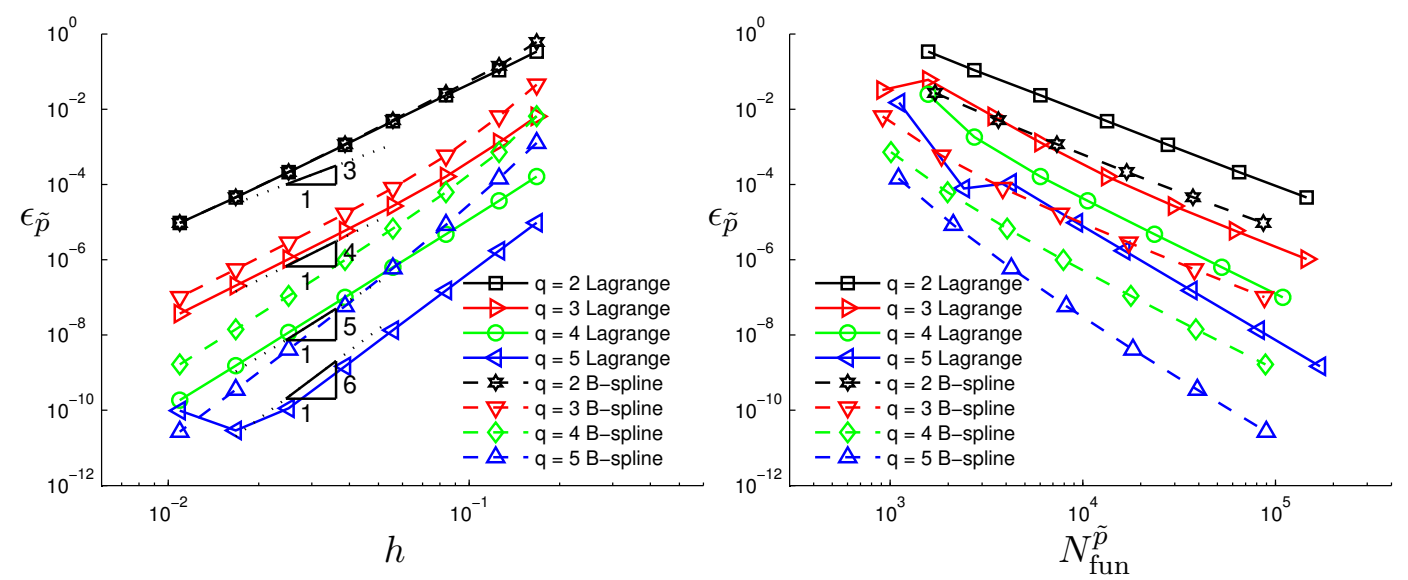

Figure 7: Error on the modulus of the computed acoustic pressure as a function of number of basis functions for the acoustic duct mode $n=3$ with frequency $f=30 \mathrm{kHz}$ using different basis function types and polynomial degrees $q$.

For the tensor-product B-spline and Lagrange discretizations with polynomial degree $q \in\{2,3,4,5\}$, we solve the problem for a range of meshes and evaluate the error using Eq. (33). We do this for the highest propagative mode $n=3$ at a frequency of $f=30 \mathrm{kHz}$. The results are shown in Fig. 7, depicting the error as a function of mesh size (a) and as a function of the number of basis functions (b). The mesh size is taken to be the largest knot span in parameter space. We note that for both the B-spline and the Lagrange discretizations, the error converges with the mesh size to 
the power of polynomial degree plus one. The slope of the error curves for any given polynomial degree is seen to be practically independent of the discretization type, both when the error is considered as a function of mesh size (a), and when the error is considered as a function of number of degrees of freedom (b). The offset of the error curves, however, is seen to change appreciably with the discretization type, and this difference increases with the polynomial degree. For a given degree and mesh size (a), the Lagrange discretization yields an error that is up to one order of magnitude smaller than the B-spline discretization. For a given degree and number of degrees of freedom (b), the B-spline discretization yields an error that is up to one order of magnitude smaller than the Lagrange discretization. These results support the appealing properties of the isogeometric method when evaluated in a per-degree-of-freedom sense as already pointed out in e.g. [23, 28]. All subsequent results are based on the B-spline-based isogeometric approach using a polynomial degree of $q=4$.

\subsection{Acoustic Truncation Boundary Conditions}

Finally, we investigate the treatment of acoustic truncation boundary conditions. We compare the mode matching formulation (MM) to a perfectly matched layer formulation (PML) in terms quality of the computed solution for the acoustic pressure, as measured by the normalised $L_{2}$-norm of the modulus of the pressure residual (33). We investigate how this error measure varies as the analysis mesh is refined by uniform knot insertions, and the number of degrees-of-freedom thereby increases, based on a quartic B-spline discretization of the acoustic pressure.

We construct a computational setup, in which we explicitly prescribe the acoustic pressure on the left-most truncation boundary $\gamma_{-}$, corresponding to the $n^{\text {th }}$ positive mode as specified in Eq. (32) for a given frequency. On the right-most truncation boundary $\gamma_{+}$, we either use the mode matching formulation, or we insert a perfectly matched layer [15, 36, 33, 37]. In the latter case, we attach a PML region of width $25 \%$ of the computational domain, let the absorption coefficient in this PML increase linearly with the spatial $x$-coordinate, and manually scale the absorption coefficient to minimize the artificial reflections.

We compare the mode matching formulation to the perfectly matched layer formulation, by investigating the error convergence for the four positive propagative modes $n \in\{0,1,2,3\}$ at the frequency $f=30 \mathrm{kHz}$. For each

combination of the two boundary conditions and the four modes, we solve the 
problem for a range of meshes, and compute the error using (33). The results

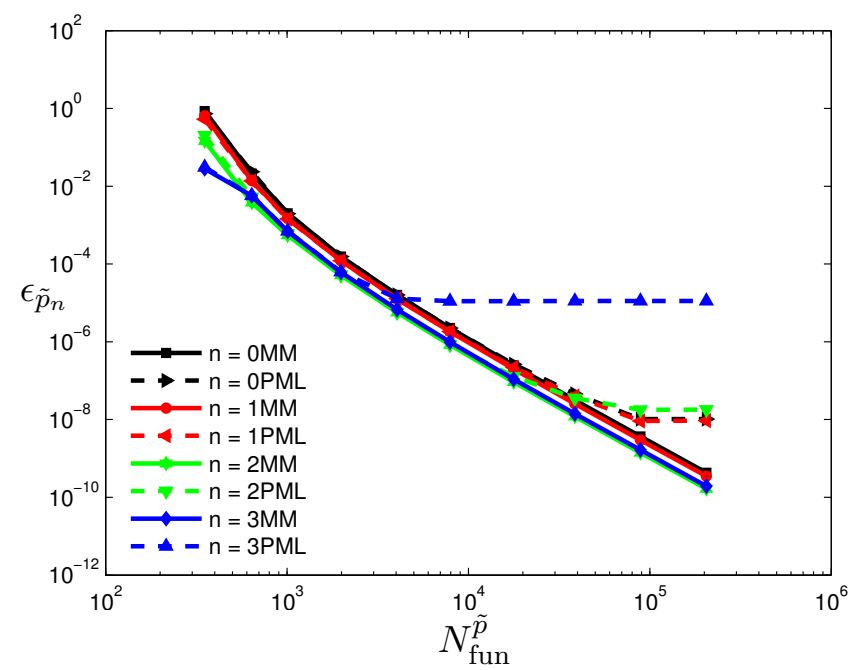

Figure 8: Error on the modulus of the computed acoustic pressure as a function of number of basis functions using a quartic B-spline discretization for different acoustic truncation boundary conditions and modes $n$.

are shown in Fig. 8. For the mode matching method, practically identical rates of convergence are found for all four modes. For the PML method, the convergence curves are quite different: the quality of the approximation seems to reach a level of saturation when the number of degrees-of-freedom is above some threshold, and the higher the mode is, the lower this threshold appears to be. We emphasize, though, that the PML results are sensitive to the PML size and absorption coefficient; Different results may be obtained through different choices.

All subsequent results are based on the mode matching formulation. The method alleviates the need for an artificial PML region, and we avoid having to choose a suitable absorption coefficient. Instead, it requires a small number of additional modal equations and unknowns on the boundary to be included, that are in general coupled to the interior equations and unknowns. For non-trivial geometries and background flows, both methods impose restrictions on the placement of the acoustic truncation boundaries, such that the computational domain has to be larger than the actual domain of interest to the acoustic analysis. For the PML method, this is due to the inclusion of the additional PML region. For the mode matching method, this is due to the requirements on the flow on the boundaries. 


\section{Numerical Experiment}

In this section we use the flow-acoustic model to compute the acoustic signal for different duct geometries, sound frequencies, and background flow speeds. Our goal is to look for a combination of duct geometry and sound frequency for which the acoustic signal is particularly sensitive to the background flow.

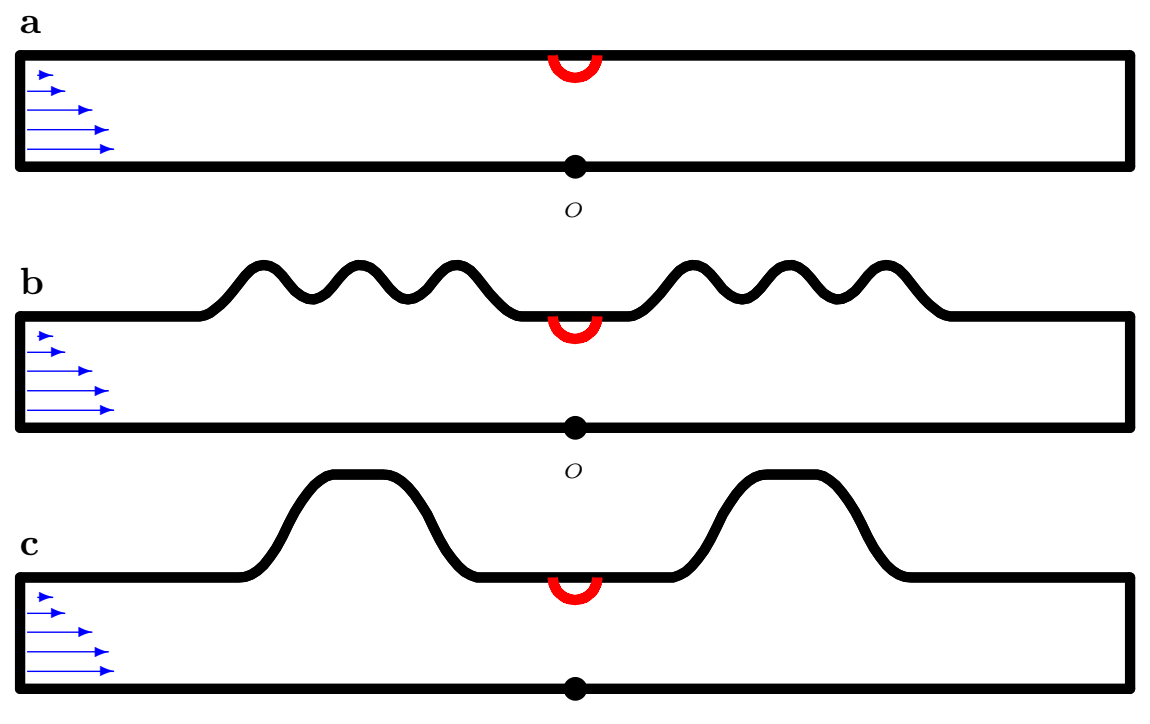

$O$

Figure 9: Design of the numerical experiment: We investigate 3 geometries $(\mathbf{a}, \mathbf{b}$, and $\mathbf{c})$, prescribe a laminar flow at the inlet (arrows), and attach an ultrasound source to the wall at the center (half circles).

The numerical experiment is sketched in Fig. 9. To assess the geometric effects, three different geometries are investigated: a straight duct (a), a duct with two shallow corrugated regions (b), and a duct with two deep bulges (c). The sound excitation is varied by assuming, for different frequencies $f$, a smooth, compactly supported acoustic source, centered at the point $(0, R)$ on the wall, of the following form:

$$
g(x, y)=g_{0} \Psi\left(x ; 0, d_{x}\right) \Psi\left(y ; r_{0}, d_{y}\right) .
$$

Here, $g_{0}$ denotes the strength of the source, $\mathbf{d}=\left(d_{x}, d_{y}\right)$ is its spatial extent, 
and the footprint function $\Psi$ is given by:

$$
\Psi(x ; a, b)=\left\{\begin{array}{ll}
e^{-\frac{1}{1-\chi^{2}}} & \text { for }|\chi|:=\left|\frac{x-a}{b}\right|<1 \\
0 & \text { otherwise }
\end{array} .\right.
$$

The flow is varied by prescribing, for different mean flow speeds $U_{0}$, a parabolic velocity profile at the inlet boundary $\gamma_{-}$:

$$
\boldsymbol{u}_{0}=\left(\frac{3}{2} U_{0}\left(1-\left(\frac{y}{R}\right)^{2}\right), 0\right)
$$

which is just the well-known Hagen-Poiseuille flow profile.

We consider again the propagation of ultrasound in a laminar flow of air through the three ducts, and use the viscosity, density, speed-of-sound, duct length, and duct inlet/outlet height summarised in Table 1. The target frequency of our studies is $f=f_{0}=30 \mathrm{kHz}$, and the parameter values for the acoustic excitation, as well as the range of variations of duct geometry, sound frequency, and flow speed are summarized in Table 2.

\begin{tabular}{llll}
\hline \hline Parameter & Description & Value & Unit \\
\hline$g_{0}$ & Source strength & $1.0 \cdot 10^{6}$ & \\
$\mathbf{d}$ & Source size & $(4.0,4.0) \cdot 10^{-3}$ & $\mathrm{~m}$ \\
$\Omega$ & Duct geometry & $\{a, b, c\}$ & \\
$f$ & Sound frequency & {$[2.75,3.25] \cdot 10^{4}$} & $\mathrm{~Hz}$ \\
$U_{0}$ & Mean flow speed & {$[0.0,1.0]$} & $\mathrm{m} / \mathrm{s}$ \\
\hline \hline
\end{tabular}

Table 2: Additional parameter values for the numerical experiment. See also Table 1.

The duct geometries are represented as a bi-quadratic spline with $26 \times 3$ control points that are adjusted from one duct to the other. The discretizations of the state variables are derived from the geometry parametrization, by increasing the polynomial degree and inserting additional knots. The number of control coefficients ranges from $291 \times 31$ (a) to $315 \times 51$ (c) for each of the background flow velocity components, $147 \times 17$ (a) and $159 \times 27$ (c) for the background flow pressure, and $292 \times 32$ (a) and $316 \times 52$ (c) for the acoustic pressure. The control net and the computational meshes for both the background flow pressure and the acoustic pressure are shown in Fig. 10 for each of the three ducts. 

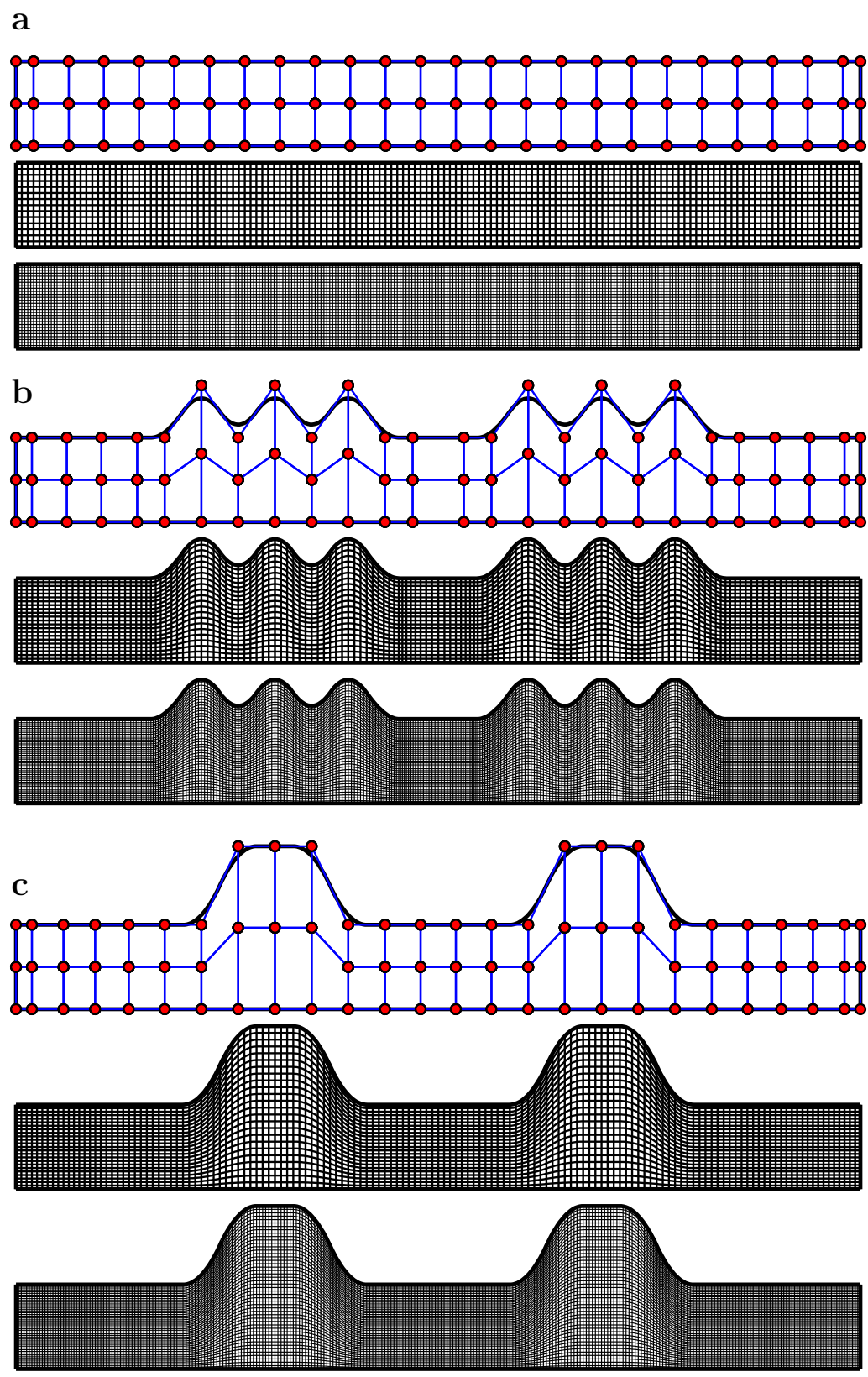

Figure 10: Control net (top), computational mesh for the background flow pressure (middle), and computational mesh for the acoustic pressure (bottom) for the straight duct (a), the corrugated duct (b), and the bulged duct (c). 
We firstly investigate the background flow. Fig. 11 depicts the flow fields in the three different ducts for the maximal flow speed $U_{0}=1 \mathrm{~m} / \mathrm{s}$. In

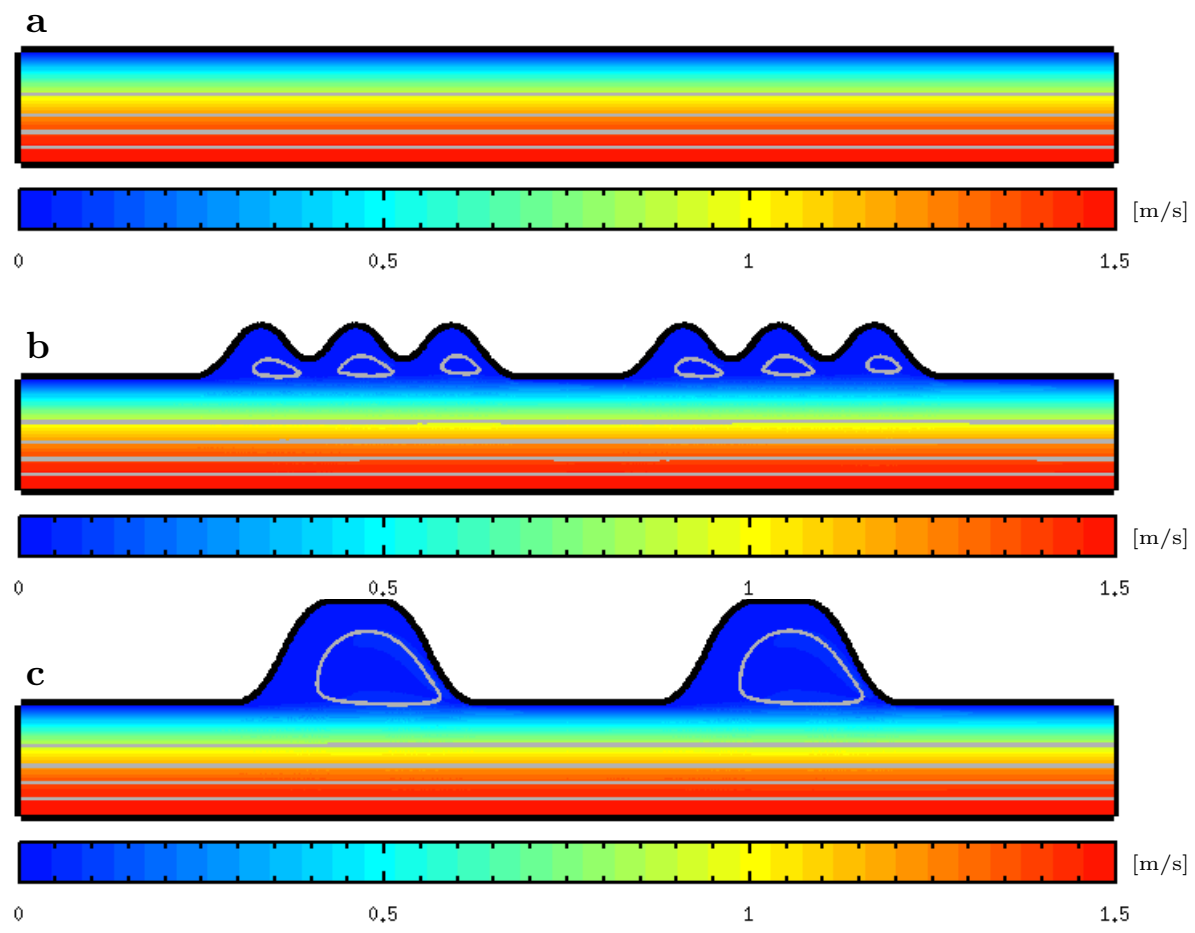

Figure 11: Flow speeds (shading) and stream lines (lines) for the mean speed $U_{0}=1 \mathrm{~m} / \mathrm{s}$ in the straight duct (a), the corrugated duct (b), and the bulged duct (c).

the straight duct (a), the parabolic velocity profile is naturally conserved down the duct. In the corrugated duct (b) and the bulged duct (c), the flow profile is perturbed in the vicinity of ondulations, where recirculating flows of different size and strength are observed.

Next, we consider the acoustic response. To this end, we note that all three duct geometries as well as the acoustic excitation are symmetric around $x=0$. Hence, asymmetries in the acoustic field around $x=0$ can arise only due to asymmetries in the background flow (acoustic reciprocity in the absence of flow). Therefore, we quantify the acoustic response for a given combination of duct geometry, sound frequency, and flow speed, by the relative 
modulus of the symmetry deviation of the acoustic pressure:

$$
\langle\delta \tilde{p}\rangle=\frac{\iint|\tilde{p}(\mathbf{x})-\tilde{p}(-\mathbf{x})| \mathrm{d} A}{\iint|\tilde{p}(\mathbf{x})| \mathrm{d} A} .
$$

Since acoustic asymmetries are caused only by background flows, this measure of asymmetry in the acoustic field is also a measure of how strongly the flow field affects the acoustic field. For reference, we also examine the mean modulus of the acoustic pressure:

$$
\langle\tilde{p}\rangle=\frac{\iint_{\Omega}|\tilde{p}(\mathbf{x})| \mathrm{d} A}{\iint_{\Omega} \mathrm{d} A} .
$$

To investigate how the acoustic response depends on the frequency for a given flow speed, we compute the relative symmetry deviation of the acoustic pressure $\langle\delta \tilde{p}\rangle$, as well as the mean acoustic pressure $\langle\tilde{p}\rangle$, for frequencies in the range $f \in[27.5,32.5] \mathrm{kHz}$ and flow speed $U_{0}=1 \mathrm{~m} / \mathrm{s}$. The results are shown in Fig. 12 for each of the three duct geometries. On the top, no significant changes in $\langle\delta \tilde{p}\rangle$ are found for the straight duct (a), while the corrugated duct (b) shows some changes with frequency. For the bulged duct (c), however, several peaks are observed, and the strongest one occurs for $f=30 \mathrm{kHz}$, where the signal experiences an increase by a factor of $\sim 10$ compared to the two other ducts. On the bottom, $\langle\tilde{p}\rangle$ shows little change in the investigated frequency range for the straight duct (a), whereas several resonance peaks are observed both for the corrugated duct (b) and the bulged duct (c). For $f=30 \mathrm{kHz}$, the bulged duct (c) displays a local maximum.

To further illustrate the phenomenon observed in the frequency sweeps above, Fig. 13 depicts the real part of the acoustic pressure field for the frequency $f=30 \mathrm{kHz}$ and the flow speed $U_{0}=1 \mathrm{~m} / \mathrm{s}$ in each of the three ducts. Both the straight duct (a) and the corrugated duct (b) exhibit high degrees of symmetry in the acoustic pressure field. In the bulged duct (c), however, the acoustic field is clearly asymmetric.

Finally, to investigate how the acoustic response depends on the flow speed for a given frequency, we compute the relative symmetry deviation of the acoustic pressure $\langle\delta \tilde{p}\rangle$, as well as the mean acoustic pressure $\langle\tilde{p}\rangle$, for flow speeds in the range $U_{0} \in[0,1] \mathrm{m} / \mathrm{s}$ and frequency $f=30 \mathrm{kHz}$. The results are shown in Fig. 14 for each of the three ducts. On the top, linear dependencies of $\langle\delta \tilde{p}\rangle$ upon $U_{0}$ are observed for the straight duct (a) and the 


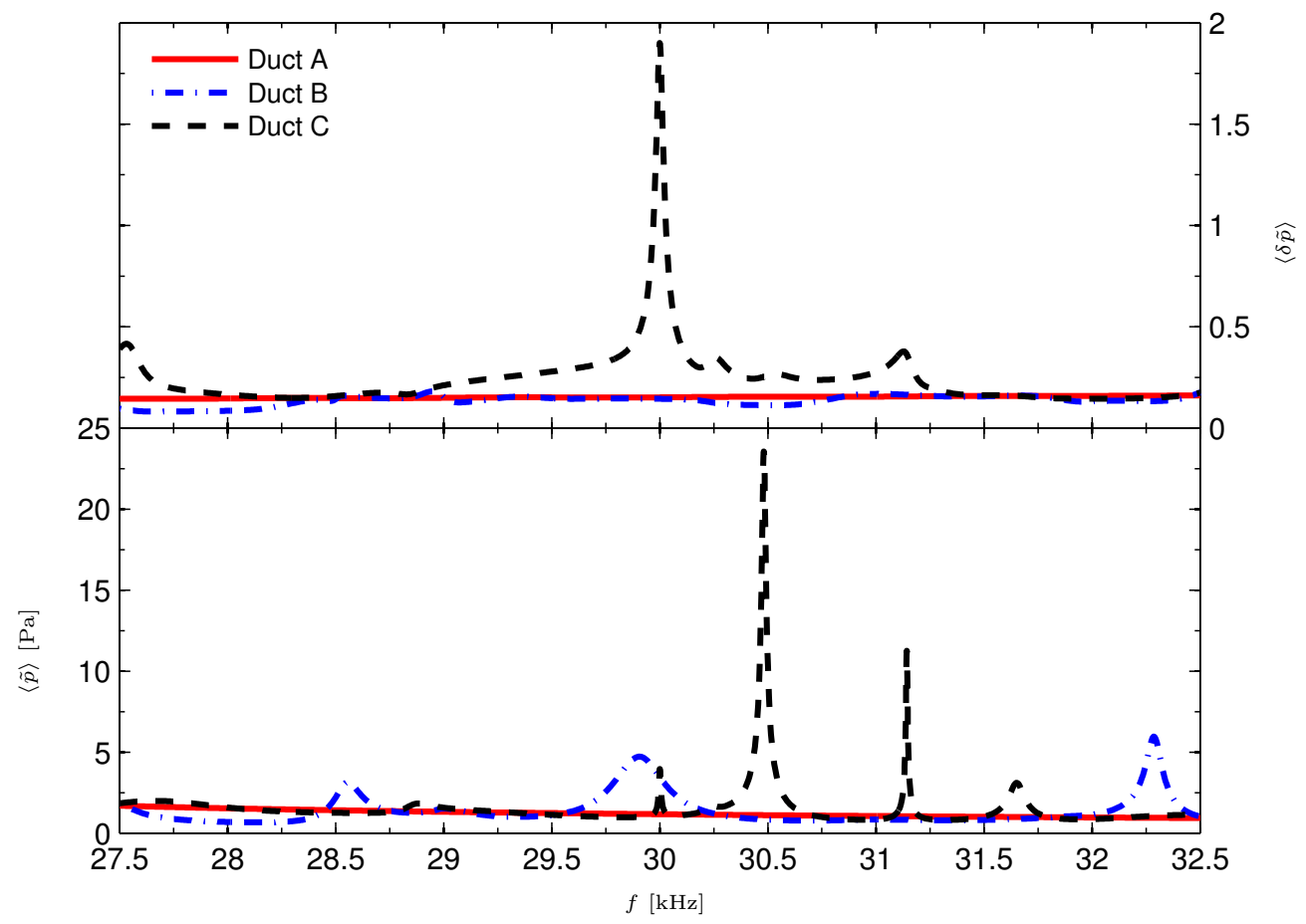

Figure 12: Relative symmetry deviation of the acoustic pressure $\langle\delta \tilde{p}\rangle$ (top) and mean acoustic pressure $\langle\tilde{p}\rangle$ (bottom) as a function of frequency $f$ for the flow speed $U_{0}=1 \mathrm{~m} / \mathrm{s}$ in the three duct geometries.

corrugated duct (b). The bulged duct (c), however, exhibits a weakly nonlinear response, in which the slope of the curve increases for $U_{0} \lesssim 0.6 \mathrm{~m} / \mathrm{s}$, and decreases for $U_{0} \gtrsim 0.6 \mathrm{~m} / \mathrm{s}$. For flow speeds $U_{0} \simeq 0.6 \mathrm{~m} / \mathrm{s}$, the slope of the curve for the bulged duct (c) is larger than that of the straight duct (a) and the corrugated duct (b) by a factor of $\sim 17$. On the bottom, constant responses in $\langle\tilde{p}\rangle$ are observed for the straight duct (a) and the corrugated duct (b), whereas a significant increase is observed for the bulged duct (c). These results clearly show that we have identified a combination of duct geometry and sound frequency, for which the sound signal is particularly sensitive to the background flow.

We conclude by noting that if more (evanescent) modes are used in the mode matching formulation for the acoustic truncation boundary conditions, by increasing $N_{m}$, practically identical results are found. If the mode matching formulation is replaced by the PML formulation described in Section 


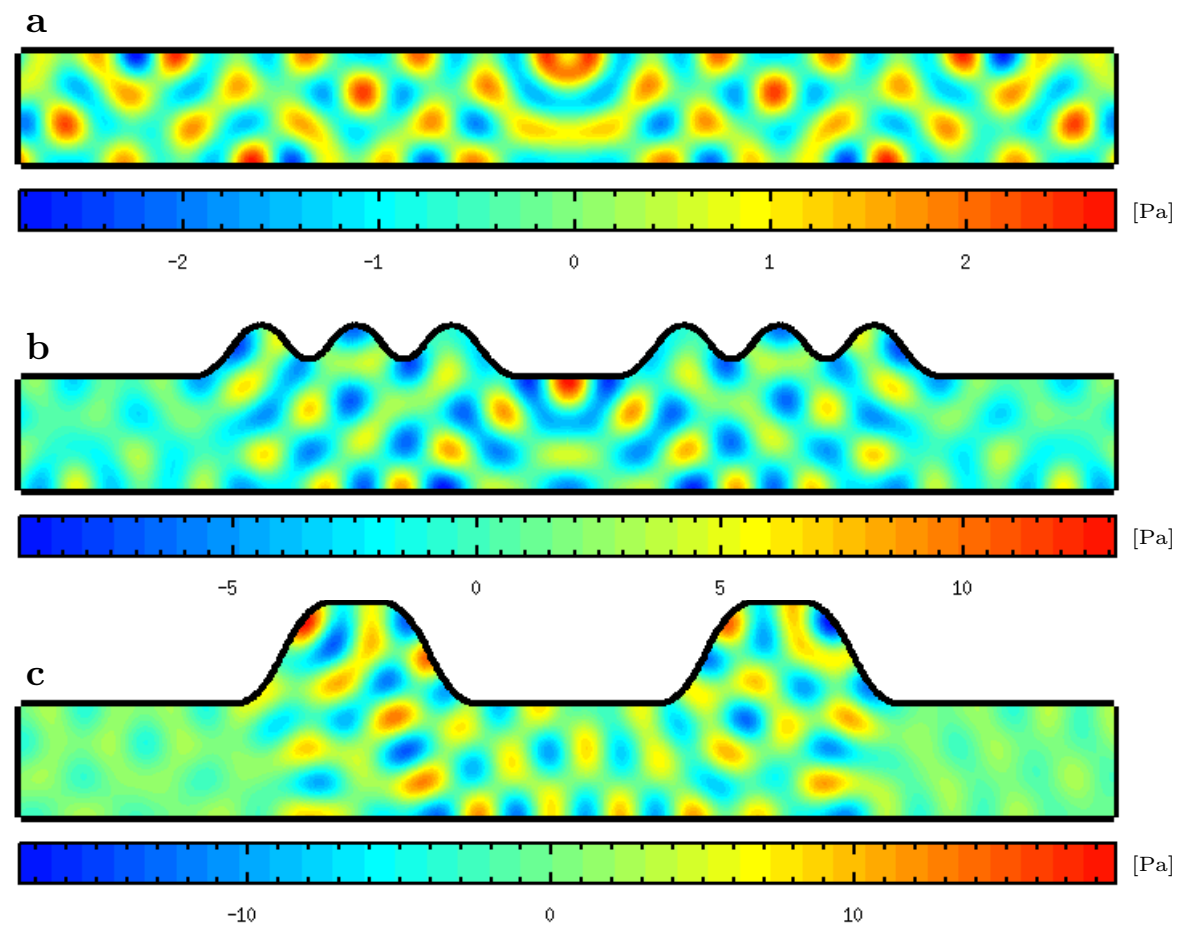

Figure 13: Real part of the acoustic pressure field $\tilde{p}$ for the frequency $f=30 \mathrm{kHz}$ and flow speed $U_{0}=1 \mathrm{~m} / \mathrm{s}$ in the straight duct $(\mathbf{a})$, the corrugated duct $(\mathbf{b})$, and the bulged duct $(\mathbf{c})$.

4.3 , equivalent results are found to within $\sim 1 \%$. If different footprints of the acoustic source are used, by changing $\mathbf{d}$, qualitatively similar results are found. In addition, unpublished numerical investigations by the authors based on Stevenson's method [38] have also shown that strong resonance couplings between flow speed and duct radius variations can occur. We emphasize, though, that the phenomenon so far only exists in numerical models and lacks experimental validation. Nevertheless, it points towards the potential importance of the geometry on flow sensitivity for acoustic wave propagation through even very slow flows. From a modeling point-of-view, future studies should include analysis and shape optimization in three dimensions to investigate and enhance the effect. 


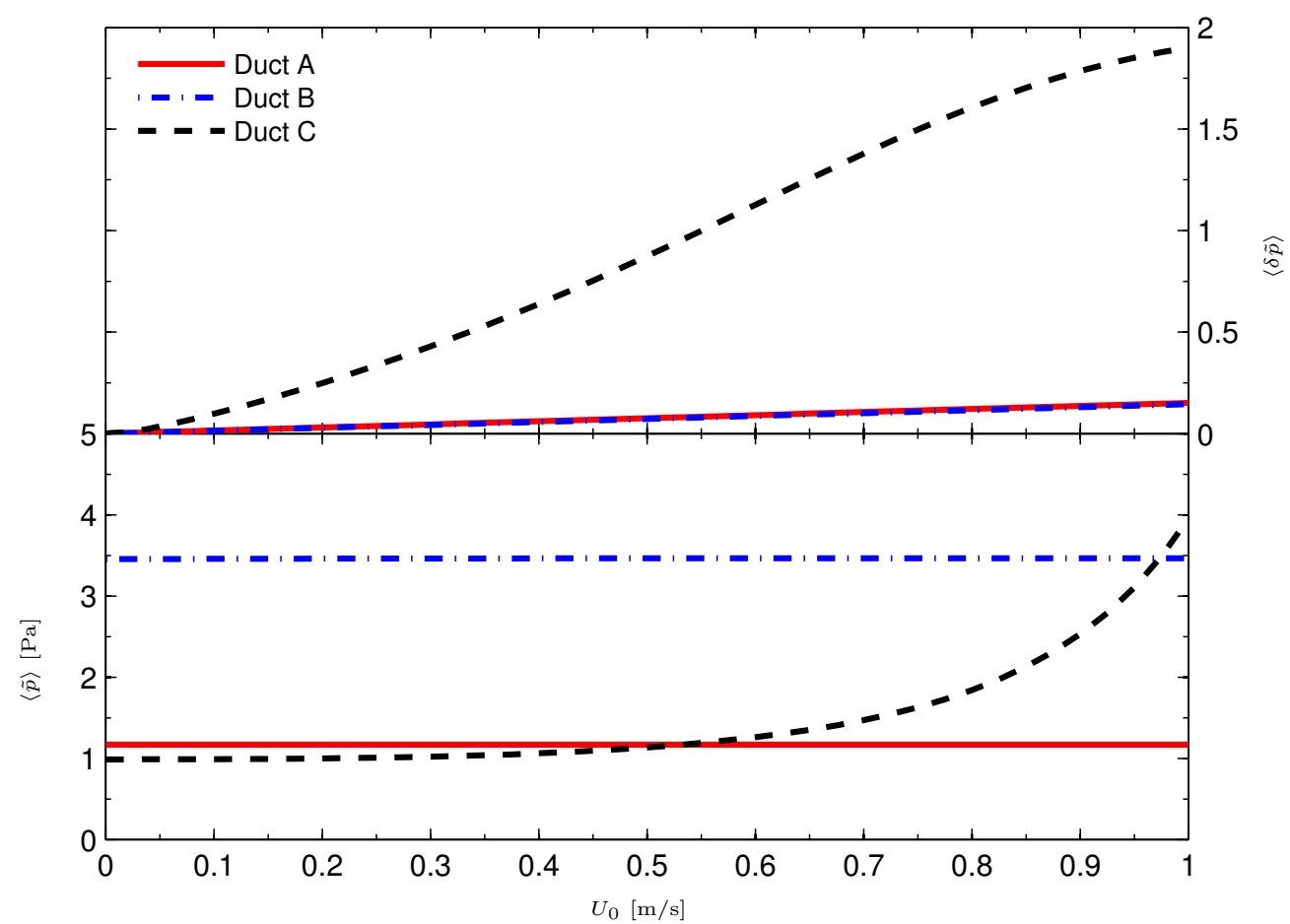

Figure 14: Relative symmetry deviation of the acoustic pressure $\langle\delta \tilde{p}\rangle$ (top) and mean acoustic pressure $\langle\tilde{p}\rangle$ (bottom) as a function of flow speed $U_{0}$ for the frequency $f=30 \mathrm{kHz}$ in the three duct geometries.

\section{Conclusions}

We have presented a flow-acoustic model of the propagation of sound through a slowly moving fluid in 2-dimensional ducts based on isogeometric analysis. The model couples the non-linear, steady state, incompressible Navier-Stokes equation in the laminar regime to a linear, time-harmonic acoustic equation in the low Mach number regime, using the solution of the former as input to the latter. B-splines are used both to model the geometry and to approximate the flow and acoustic fields. Acoustic boundary conditions along artificial truncation boundaries are treated by matching acoustic modes. The model has been validated against known acoustic modes for a uniform flow through a straight duct. These tests clearly supported the applicability of the method, and in particular demonstrated desireable error convergence properties for higher order polynomial approximations of the acoustic pressure, that are embedded in isogeometric analysis. Using 
the model, acoustic signal changes vs. duct geometry have been examined as a function of frequency and background flow, facilitated by the geometric flexibility of isogometric analysis. A combination of duct geometry and sound frequency was identified for which the acoustic signal is particularly sensitive to the background flow. This enhanced sensitivity deserves closer examination in future studies.

\section{Appendix A. Derivation of the Acoustic Equation}

In this section, we derive Eq. (8). To shorten the notation, we let $\partial_{t}:=$ $\partial / \partial t$ denote differentiation with respect to time, and $\partial_{x}:=\partial / \partial x$ and $\partial_{y}:=$ $\partial / \partial y$ differentiation with respect to spatial coordinates.

We set out from the Navier-Stokes and mass conservation equations (1), and start by assuming that we may neglect viscous effects for the acoustic signal (isentropic sound propagation). The governing equations (1) then read:

$$
\begin{array}{r}
\rho \partial_{t} \mathbf{u}+\rho(\mathbf{u} \cdot \nabla) \mathbf{u}+\nabla p=\mathbf{0} \\
\partial_{t} \rho+\nabla \cdot(\rho \mathbf{u})=0 .
\end{array}
$$

Next, we assume that the background flow $\left(\mathbf{u}_{0}, p_{0}, \rho_{0}\right)$ fulfills the mass conservation equation and Navier-Stokes equations including viscous effects, that it is incompressible, i.e., $\partial_{t} \rho_{0}=\partial_{x} \rho_{0}=\partial_{y} \rho_{0}=0$, and hence $\nabla \cdot \mathbf{u}_{0}=0$ by Eq. (A.1b), and that it is stationary, i.e. $\partial_{t} \mathbf{u}_{0}=\mathbf{0}$, as in the flow model in Section 3.3 above. Inserting the conventions (2) into the governing equations (A.1), while using the above, neglecting second orders terms in the acoustic disturbances $\left(\mathbf{u}^{\prime}, p^{\prime}, \rho^{\prime}\right)$ first-order terms in $\mathbf{u}^{\prime}$ multiplied by small secondorder terms in the background flow, we find:

$$
\begin{aligned}
\rho_{0} \partial_{t} \mathbf{u}^{\prime}+\rho_{0}\left(\mathbf{u}_{0} \cdot \nabla\right) \mathbf{u}^{\prime}+\rho_{0}\left(\mathbf{u}^{\prime} \cdot \nabla\right) \mathbf{u}_{0}+\nabla p^{\prime} & =\mathbf{0}, \\
\partial_{t} \rho^{\prime}+\mathbf{u}_{0} \cdot \nabla \rho^{\prime}+\rho_{0} \nabla \cdot \mathbf{u}^{\prime} & =0 .
\end{aligned}
$$

Dividing Eq. (A.2a) by $\rho_{0}$, multiplying Eq. (A.2b) by $c^{2}$, and utilizing the acoustic relation $\frac{D p^{\prime}}{D t}=c^{2} \frac{D \rho^{\prime}}{D t}$, where $c$ is the speed of sound in the fluid, we obtain:

$$
\begin{array}{r}
\partial_{t} \mathbf{u}^{\prime}+\left(\mathbf{u}_{0} \cdot \nabla\right) \mathbf{u}^{\prime}+\left(\mathbf{u}^{\prime} \cdot \nabla\right) \mathbf{u}_{0}+\frac{1}{\rho_{0}} \nabla p^{\prime} \\
=\mathbf{0}, \\
\partial_{t} p^{\prime}+\mathbf{u}_{0} \cdot \nabla p^{\prime}+\rho_{0} c^{2} \nabla \cdot \mathbf{u}^{\prime}=0 .
\end{array}
$$


Next, we apply separation-of-variables and assume time-harmonic conditions. We seek acoustic solutions $\mathbf{u}^{\prime}$ and $p^{\prime}$ to Eqs. (A.3) of the following form:

$$
\mathbf{u}^{\prime}(t, x, y)=e^{-i \omega t} \tilde{\mathbf{u}}(x, y), \quad p^{\prime}(t, x, y)=e^{-i \omega t} \tilde{p}(x, y) .
$$

where $\omega$ denotes the acoustic angular frequency. Inserting these relations into Eqs. (A.3), ignoring the second order term in the background flow $\mathbf{u}_{0}$ in Eq. (A.3a), and dividing by the common temporal part $e^{-i \omega t}$, we obtain the following equations in the spatial parts of the acoustic fields:

$$
\begin{aligned}
-i \omega \tilde{\mathbf{u}}+\left(\mathbf{u}_{0} \cdot \nabla\right) \tilde{\mathbf{u}}+(\tilde{\mathbf{u}} \cdot \nabla) \mathbf{u}_{0}+\frac{1}{\rho_{0}} \nabla \tilde{p} & =\mathbf{0}, \\
-i \omega \tilde{p}+\mathbf{u}_{0} \cdot \nabla \tilde{p}+\rho_{0} c^{2} \nabla \cdot \tilde{\mathbf{u}} & =0 .
\end{aligned}
$$

To reduce this system, Eq. (A.5a) gives us:

$$
\tilde{\mathbf{u}}=-\frac{i}{\omega}\left(\frac{1}{\rho_{0}} \nabla \tilde{p}+(\tilde{\mathbf{u}} \cdot \nabla) \mathbf{u}_{0}+\left(\mathbf{u}_{0} \cdot \nabla\right) \tilde{\mathbf{u}}\right)=-\frac{i}{\omega \rho_{0}} \nabla \tilde{p}+\mathcal{O}\left(\mathbf{u}_{0}\right),
$$

and by inserting this into Eq. (A.5b), we find:

$$
-i \omega \tilde{p}+\mathbf{u}_{0} \cdot \nabla \tilde{p}-\frac{i \rho_{0} c^{2}}{\omega} \nabla \cdot\left(\frac{1}{\rho_{0}} \nabla \tilde{p}+(\tilde{\mathbf{u}} \cdot \nabla) \mathbf{u}_{0}+\left(\mathbf{u}_{0} \cdot \nabla\right) \tilde{\mathbf{u}}\right)=0 .
$$

Next, we neglect all terms quadratic or of higher order in the background flow $\mathbf{u}_{0}$ and its derivatives, assuming low Mach numbers, i.e. $\left\|\mathbf{u}_{0}\right\| \ll c$. Exploiting again Eq. (A.6) in the above, we then obtain the following equation in the spatial part of acoustic pressure only:

$$
-i \omega \tilde{p}+\mathbf{u}_{0} \cdot \nabla \tilde{p}-\frac{i c^{2}}{\omega} \nabla^{2} \tilde{p}-\frac{c^{2}}{\omega^{2}} \nabla \cdot\left((\nabla \tilde{p} \cdot \nabla) \mathbf{u}_{0}+\left(\mathbf{u}_{0} \cdot \nabla\right) \nabla \tilde{p}\right)=0 .
$$

To simplify Eq. (A.8), we use the following identity for two arbitrary vector functions $\mathbf{a}=\left(a_{1}, a_{2}\right)$ and $\mathbf{b}=\left(b_{1}, b_{2}\right)$ :

$$
\begin{aligned}
& \nabla \cdot((\mathbf{b} \cdot \nabla) \mathbf{a}+(\mathbf{a} \cdot \nabla) \mathbf{b})= \\
& 2\left(\partial_{x} \mathbf{a} \cdot \nabla b_{1}+\partial_{y} \mathbf{a} \cdot \nabla b_{2}\right)+(\mathbf{a} \cdot \nabla)(\nabla \cdot \mathbf{b})+(\mathbf{b} \cdot \nabla)(\nabla \cdot \mathbf{a}),
\end{aligned}
$$

which may be verified by straightforward calculations. Taking $\mathbf{a}=\mathbf{u}_{0}$ and $\mathbf{b}=\nabla \tilde{p}$ in the above yields:

$$
\begin{aligned}
& \nabla \cdot\left((\nabla \tilde{p} \cdot \nabla) \mathbf{u}_{0}+\left(\mathbf{u}_{0} \cdot \nabla\right) \nabla \tilde{p}\right)= \\
& 2\left(\partial_{x} \mathbf{u}_{0} \cdot \nabla \partial_{x} \tilde{p}+\partial_{y} \mathbf{u}_{0} \cdot \nabla \partial_{y} \tilde{p}\right)+\left(\mathbf{u}_{0} \cdot \nabla\right)\left(\nabla^{2} \tilde{p}\right)+(\nabla \tilde{p} \cdot \nabla)\left(\nabla \cdot \mathbf{u}_{0}\right) .
\end{aligned}
$$


Now, taking the divergence of (A.6), and inserting this into (A.5b), we find:

$$
\nabla^{2} \tilde{p}=-\frac{w^{2}}{c^{2}} p+\mathcal{O}\left(\mathbf{u}_{0}\right)
$$

Next, we may insert Eq. (A.10) into Eq. (A.8), using the above relation, neglecting again quadratic or higher order terms in the background flow $\mathbf{u}_{0}$ and its derivatives, and exploiting the incompressibility of the background flow $\nabla \cdot \mathbf{u}_{0}=0$. After some manipulations we find:

$$
\nabla^{2} \tilde{p}+\frac{\omega^{2}}{c^{2}} \tilde{p}+\frac{2 i}{\omega}\left(\frac{\omega^{2}}{c^{2}} \mathbf{u}_{0} \cdot \nabla \tilde{p}-\partial_{x} \mathbf{u}_{0} \cdot \nabla \partial_{x} \tilde{p}-\partial_{y} \mathbf{u}_{0} \cdot \nabla \partial_{y} \tilde{p}\right)=0 .
$$

Finally, we may define the wave number $k:=\omega / c$, and introduce an acoustic source $g$ on the right hand side. This gives us:

$$
\nabla^{2} \tilde{p}+k^{2} \tilde{p}+\frac{2 i}{\omega}\left(k^{2} \mathbf{u}_{0} \cdot \nabla \tilde{p}-\frac{\partial \mathbf{u}_{0}}{\partial x} \cdot \nabla \frac{\partial \tilde{p}}{\partial x}-\frac{\partial \mathbf{u}_{0}}{\partial y} \cdot \nabla \frac{\partial \tilde{p}}{\partial y}\right)=g .
$$

\section{References}

[1] P. Exner, P. Seba, Bound states in curved quantum waveguides, Journal of Mathematical Physics 30 (1989) 2574-2580.

[2] F. H. Borgnis, C. H. Papas, Electromagnetic waveguides and resonators, in: S. Flugge (Ed.), Encyclopedia of Physics, volume 16, SpringerVerlag, 1958, p. 326.

[3] J. Goldstone, R. L. Jaffe, Bound states in twisting tubes, Physical Review B 45 (1992) 14100-14107.

[4] P. W. Anderson, Absence of diffusion in certain random lattices, Physical Review 109 (1958) 1492-1505.

[5] N. E. Hurt, Mathematical Physics of Quantum Wires and Devices. From Spectral Resonances to Anderson Localization, Mathematics and Its Applications, Kluwer Academic Publisher, 2000.

[6] V. I. Grinchenko, Wave motion localization effects in elastic waveguides, International Applied Mechanics 41 (2005) 988-994.

[7] J. D. Joannopoulos, R. D. Meade, J. N. Winn, Photonic Crystals: Molding the flow of light, Princeton University Press, 1995. 
[8] M. M. Sigalas, E. N. Economou, Band structure of elastic waves in two dimensional systems, Solid State Communications 86 (1993) 141-143.

[9] M. S. Kushwaha, G. Martinez, Photonic bandgaps in two-dimensional semiconductor-dielectric composite crystals, Journal of the Optical Society of America B 23 (2006) pp. 1460-1470.

[10] A. A. Krokhin, J. Arriaga, L. N. Gumen, Speed of sound in periodic elastic composites, Physical Review Letters 91 (2003) 264302 (4 pages).

[11] L. C. L. Y. Voon, M. Willatzen, Electron states in modulated nanowires, Journal of Applied Physics 93 (2003). Also published in virtual Journal of Nanoscale Science \& Technology, June 9, 2003, Volume 7, Issue 23.

[12] V. Pagneux, N. Amir, J. Kergomard, A study of wave propagation in varying cross-section waveguides by modal decomposition. part i. theory and validation, Journal of the Acoustical Society of America 100 (1996) 2034-2048.

[13] E. Brambley, N. Peake, Sound transmission in strongly curved slowly varying cylindrical ducts with flow, Journal of Fluid Mechanics 596 (2008) 387-412.

[14] S. Rienstra, Sound propagation in slowly varying lined flow ducts of arbitrary cross-section, Journal of Fluid Mechanics 495 (2003) 157-173.

[15] E. Becache, A.-S. B.-B. Dhia, G. Legendre, Perfectly matched layers for the convected Helmholtz equation, SIAM Journal on Numerical Analysis 42 (2004) 409-433.

[16] E. Redon, A.-S. B.-B. Dhia, J.-F. Mercier, S. P. Pari, Non-reflecting boundary conditions for acoustic propagation in ducts with acoustic treatment and mean flow, International Journal for Numerical Methods in Engineering (2011).

[17] R. J. Astley, W. Eversman, Acoustic transmission in non-uniform ducts with mean flow, part II: the finite element method, Journal of Sound and Vibration 74 (1981) 103-121.

[18] C. Peyret, G. Élias, Finite-element method to study harmonic aeroacoustics problems, Journal of the Acoustical Society of America 110 (2001) 661-668. 
[19] A.-S. B.-B. Dhia, E.-M. Duclairoir, G. Legendre, J.-F. Mercier, Timeharmonic acoustic propagation in the presence of a shear flow, Journal of Computational and Applied Mathematics 204 (2007) 428-439.

[20] A.-S. B.-B. Dhia, J.-F. Mercier, F. Millot, S. Pernet, A low-Mach number model for time-harmonic acoustics in arbitrary flows, Journal of Computational and Applied Mathematics 234 (2010) 1868-1875.

[21] T. J. R. Hughes, J. A. Cottrell, Y. Bazilevs, Isogeometric analysis: CAD, finite elements, NURBS, exact geometry and mesh refinement, Computer methods in applied mechanics and engineering 194 (2005) 4135-4195.

[22] J. A. Cottrell, T. J. R. Hughes, Y. Bazilevs, Isogeometric Analysis: Toward Integration of CAD and FEA, John Wiley and Sons, 2009.

[23] J. A. Cottrell, A. Reali, Y. Bazilevs, T. J. R. Hughes, Isogeometric analysis of structural vibrations, Computer methods in applied mechanics and engineering 195 (2006) 5257-5296.

[24] Y. Bazilevs, T. J. R. Hughes, NURBS-based isogeometric analysis for the computation of flows about rotating components, Computational Mechanics 43 (2008) 143-150.

[25] Y. Bazilevs, I. Akkerman, Large eddy simulation of turbulent taylorcouette flow using isogeometric analysis and the residual-based variational multiscale method, Journal of Computational Physics 229 (2010) 3402-3414.

[26] A. Buffa, C. de Falco, G. Sangalli, Isogeometric Analysis: Stable elements for the 2D Stokes equation, International Journal for Numerical Methods in Fluids (2011).

[27] P. N. Nielsen, A. R. Gersborg, J. Gravesen, N. L. Pedersen, Discretizations in isogeometric analysis of Navier-Stokes flow, Computer methods in applied mechanics and engineering 200 (2011) 3242-3253.

[28] T. J. R. Hughes, A. Reali, G. Sangalli, Duality and unified analysis of discrete approximations in structural dynamics and wave propagation: Comparison of $p$-method finite elements with $k$-method NURBS, 
Computer methods in applied mechanics and engineering 197 (2008) 4104-4124.

[29] L. Dedè, C. Jäggli, A. Quarteroni, Isogeometric numerical dispersion analysis for two-dimensional elastic wave propagation, Computer Methods in Applied Mechanics and Engineering 284 (2015) 320-348.

[30] Y. Bazilevs, I. Akkerman, D. J. Benson, G. Scovazzi, M. J. Shashkov, Isogeometric analysis of Lagrangian hydrodynamics, Journal of Computational Physics 243 (2013) 224-243.

[31] R. Simpson, M. Scott, M. Taus, D. Thomas, H. Lian, Acoustic isogeometric boundary element analysis, Computer methods in applied mechanics and engineering (2013). DOI: http://dx.doi.org/10.1016/j. cma.2013.10.026.

[32] D. C. Pridmore-Brown, Sound propagation in a fluid through an attenuating duct, Journal of Fluid Mechanics 4 (1958) 393-406.

[33] D. Givoli, Computational absorbing boundaries, in: S. Marburg, B. Nolte (Eds.), Computational Acoustics Of Noise Propagation In Fluids - Finite And Boundary Element Methods, Springer, 2008, pp. 145166.

[34] R. Astley, FE mode-matching schemes for the exterior Helmholtz problem and their relationship to the FE-DtN approach, Communications in numerical methods in engineering 12 (1996) 257-267.

[35] L. Piegl, W. Tiller, The NURBS Book, Springer, 1995.

[36] E. Becache, A.-S. B.-B. Dhia, G. Legendre, Perfectly matched layers for time-harmonic acoustics in the presence of a uniform flow, SIAM Journal on Numerical Analysis 44 (2006) 1191-1217.

[37] A. Bermúdez, L. Hervella-Nieto, A. Prieto, R. Rodríguez, Perfectly Matched Layers, in: S. Marburg, B. Nolte (Eds.), Computational Acoustics Of Noise Propagation In Fluids - Finite And Boundary Element Methods, Springer, 2008, pp. 467-196.

[38] A. F. Stevenson, Exact and approximate equations for wave propagation in acoustic horns, Journal of Applied Physics 22 (1951) 1461-1463. 